\title{
Simplifying Schema Mappings
}

\author{
Diego Calvanese \\ KRDB Research Centre \\ Free Univ. of Bozen-Bolzano \\ I-39100 Bolzano, Italy \\ calvanese@inf.unibz.it
}

\author{
Giuseppe De Giacomo \\ Maurizio Lenzerini \\ Dip. di Inf. e Sist. \\ Univ. di Roma "La Sapienza" \\ I-00185 Roma, Italy \\ lastname@dis.uniroma1.it
}

\author{
Moshe Y. Vardi \\ Dep. of Computer Science \\ Rice University, P.O. Box 1892 \\ Houston, TX 77251-1892, \\ U.S.A. \\ vardi@cs.rice.edu
}

\begin{abstract}
A schema mapping is a formal specification of the relationship holding between the databases conforming to two given schemas, called source and target, respectively. While in the general case a schema mapping is specified in terms of assertions relating two queries in some given language, various simplified forms of mappings, in particular LAV and GAV, have been considered, based on desirable properties that these forms enjoy. Recent works propose methods for transforming schema mappings to logically equivalent ones of a simplified form. In many cases, this transformation is impossible, and one might be interested in finding simplifications based on a weaker notion, namely logical implication, rather than equivalence. More precisely, given a schema mapping $M$, find a simplified (LAV, or GAV) schema mapping $M^{\prime}$ such that $M^{\prime}$ logically implies $M$. In this paper we formally introduce this problem, and study it in a variety of cases, providing techniques and complexity bounds. The various cases we consider depend on three parameters: the simplified form to achieve (LAV, or GAV), the type of schema mapping considered (sound, or exact), and the query language used in the schema mapping specification (conjunctive queries and variants over relational databases, or regular path queries and variants over graph databases). Notably, this is the first work on comparing schema mappings for graph databases.
\end{abstract}

\section{Categories and Subject Descriptors}

D.2.12 [Software Engineering]: Interoperability-data mapping; H.2.3 [Database Management]: Languagesquery languages

\section{General Terms}

Theory

\section{Keywords}

Schema mappings, GAV, LAV, graph databases, conjunctive

Permission to make digital or hard copies of all or part of this work for personal or classroom use is granted without fee provided that copies are not made or distributed for profit or commercial advantage and that copies bear this notice and the full citation on the first page. To copy otherwise, to republish, to post on servers or to redistribute to lists, requires prior specific permission and/or a fee.

ICDT 2011, March 21-23, 2011, Uppsala, Sweden.

Copyright 2011 ACM 978-1-4503-0529-7/11/0003 ...\$10.00. queries

\section{INTRODUCTION}

A schema mapping is a formal specification of the relationship holding between the databases conforming to two given schemas. Many papers point out the importance of schema mappings in several data management tasks, especially those requiring inter-operability between different information systems, such as data integration [38, 36], data exchange [37, 14], and model management [17].

In data integration, schema mappings are established between the source schema and the global (mediated) schema, and are used to decide how to access the source data for answering queries posed in terms of the global schema. In data exchange, schema mappings are specified in terms of a source schema and a target schema, and determine how the source data should be transferred to the target in order to populate a database conforming to the target schema. Schema mappings are also the main objects of interest in model management, whose goal is to support the creation, compilation, reuse, evolution, and execution of mappings between schemas, expressed in a wide range of model.

A schema mapping is constituted by two schemas and a set of mapping assertions between the two. We follow the data exchange terminology, and call the two schema source and target, respectively. As usual, we assume that each assertion relates a query $q_{s}$ over the source to a query $q_{t}$ over the target, and specifies a correspondence between the tuples computed by $q_{s}$ in source databases and those computed by $q_{t}$ in target databases. In the following, we consider two types of schema mappings, called sound and exact, respectively. The correspondence specified by assertions in a sound mapping is inclusion, whereas the correspondence specified by assertions in an exact mapping is equality. Semantically, a schema mapping $M$ is characterized by the set of pairs $\left(\mathcal{D}_{s}, \mathcal{D}_{t}\right)$ of databases such that $\mathcal{D}_{s}$ is a source database, $\mathcal{D}_{t}$ is a target database, and they satisfy the correspondences sanctioned by the assertions in $M$.

Since the pioneering work on schema mappings in data integration [46], various restricted forms of mappings have been considered, in particular LAV and GAV. In LAV (Local-As-Views) mappings, the source queries in the assertions are constituted by one atom, and exactly one assertion appears for each relation symbol in the source schema. In other words, a LAV mapping associates to each element of the source schema one view over the target schema. Conversely, a GAV (Global-As-Views) mapping associates to each element of the target schema one view over the source 
schema. Extending the above terminology, the term GLAV is often used to refer to unrestricted forms of schema mappings.

Schema mappings have been widely investigated in the last years. In $[26,28,7,40]$ the emphasis is on providing foundations for data exchange systems based on schema mappings. Other works deal with answering queries posed to the target schema on the basis of both the data at the sources, and a set of source-to-target mapping assertions (see, for instance, $[3,7]$ and the surveys in $[46,36]$ ). A large body of work has been devoted to studying operators on schema mappings relevant to model management, notably, composition, merge, and inverse (see, for example [41, 30, $31,29,11,12,8,10])$.

Recently, there has been a growing interest in principles and tools for comparing both schema mapping languages, and schema mappings expressed in a certain language. Comparing schema mapping languages aims at characterizing such languages in terms of both expressive power, and complexity of mapping-based computational tasks $[45,6]$. In particular, [45] studies various relational schema mapping languages with the goal of characterizing them in terms of structural properties possessed by the schema mappings specified in these languages.

Methods for comparing schema mappings have been recently proposed in $[27,34,29,8]$. In [29, 8], schema mappings are compared with respect to their ability to transfer source data and avoid redundancy in the target databases, as well as their ability to cover target data. More relevant to the present paper is the work in [27], which introduces three notions of equivalence. The first one is the usual notion based on logic: two schema mappings are logically equivalent if they are indistinguishable by the semantics, i.e., if they are satisfied by the same set of database pairs. The other two notions, called data exchange and conjunctive query equivalence, respectively, are relaxations of logical equivalence, capturing indistinguishability for different purposes. In [34], schema mapping optimization is studied, based on logical equivalence. In particular, a set of optimality criteria are proposed for an important class of relational schema mappings, and rewriting rules for transforming a schema mapping into an equivalent optimal one are presented. Notably, LAV and GAV enjoy many of the optimality criteria mentioned in the paper. It follows that the proposed rewriting rules often lead to transforming an input schema mapping into one of the two simplified forms.

The above discussion shows that the work on optimization and simplification of schema mappings has concentrated so far on equivalence preserving transformations. However, there are cases where equivalence preserving simplification is not possible, as demonstrated for LAV by the following example.

Example 1. Consider the schema mapping $M$ constituted by the following mapping assertion

$$
\begin{aligned}
&\left\{(x, w, z) \mid r_{1}(x, w, y) \wedge r_{2}(y, z)\right\} \sim \\
&\left\{(x, w, z) \mid t_{1}(x, w, v) \wedge t_{1}(v, w, e) \wedge t_{2}(e, z)\right\}
\end{aligned}
$$

Suppose that $M$ is interpreted under the sound semantics. Now, let $\mathcal{D}_{s}$ be a database such that $r_{1}^{\mathcal{D}_{s}} \neq \emptyset$, and $r_{2}^{\mathcal{D}_{s}}=\emptyset$, and let $\mathcal{D}_{t}$ be the empty database. Clearly, $\left(\mathcal{D}_{s}, \mathcal{D}_{t}\right) \models M$. On the other hand, for any sound LAV mapping $M^{\prime}$ on the same alphabet as $M$, it holds that $\left(\mathcal{D}_{s}, \mathcal{D}_{t}\right) \not \models M^{\prime}$, because $r_{1}^{\mathcal{D}_{s}} \neq \emptyset$, while the query $M^{\prime}\left(r_{1}\right)$ that $M^{\prime}$ associates to $r_{1}$ is such that $M^{\prime}\left(r_{1}\right)^{\mathcal{D}_{t}}=\emptyset$, and therefore the assertion $r_{1} \leadsto$ $M^{\prime}\left(r_{1}\right)$ in $M^{\prime}$ cannot be satisfied by $\left(\mathcal{D}_{s}, \mathcal{D}_{t}\right)$. It follows that no LAV mapping $M^{\prime}$ exists such that $M \models M^{\prime}$, and therefore equivalence preserving LAV simplification of $M$ is impossible to achieve.

To address such cases, we argue that simplification should be based on a weaker notion, namely logical implication, rather than equivalence.

ExAmple 2. Refer again to the schema mapping $M$ of Example 1, and consider the sound LAV mapping $M^{\prime \prime}$ constituted by the following two mapping assertions:

$$
\begin{aligned}
\left\{(x, w, y) \mid r_{1}(x, w, y)\right\} & \leadsto\left\{(x, w, y) \mid t_{1}(x, w, v) \wedge t_{1}(v, w, y)\right\} \\
\left\{(x, y) \mid r_{2}(x, y)\right\} & \leadsto\left\{(x, y) \mid t_{2}(x, y)\right\}
\end{aligned}
$$

It is not difficult to see that $M^{\prime \prime} \models M$. Therefore, if we are happy with LAV simplification based on logical implication rather than logical equivalence, $M^{\prime \prime}$ represents an acceptable simplification of $M$.

Mapping simplification based on logical implication is the subject of this paper. The problem can be stated as follows: given a schema mapping $M$, check whether a simplified (LAV, or GAV) schema mapping $M^{\prime}$ exists such that $M^{\prime}$ logically implies $M$ (and, if it exists, find one). We formally introduce this problem, and study it in a variety of cases, depending on three parameters:

1. the simplified form to achieve (LAV, or GAV),

2. the type of schema mapping considered (sound, or exact), and

3. the data model and the query language used in the schema mapping specification.

As for the first parameter, we essentially concentrate on LAV in this paper. We discuss GAV only briefly, pointing out that GAV simplification is an open problem in several cases.

As for the type of mapping, although the sound semantics is the more popular one in data exchange [37], the importance of considering exact schema mappings is widely recognized for both data exchange [33], and data integration [35].

As for the data model and the query language used in schema mappings, we consider both the relational data model with conjunctive queries and unions thereof, and the graph database model with regular path queries and their extensions. Note that, while schema mappings have been extensively studied for relational data, and, to some extent, for XML data [9], this is the first paper on comparing schema mappings for graph databases. Graph databases [25] were introduced in the ' $80 \mathrm{~s}$, and are regaining wide attention recently [2, 32, 15], for their relevance in areas such as semi-structured data, biological data management, social networks, and the semantic web.

The results we present in this paper can be summarized as follows. We first illustrate our ideas with relational mappings, where the results follow fairly easily from the characterization of containment for conjunctive queries and unions thereof. We show that LAV simplification is NP-complete in the case of both sound and exact schema mappings based on conjunctive queries. In the case of unions of conjunctive queries, the problem is still in NP for sound mappings, while it is in $\Pi_{2}^{p}$ for exact ones. 
For graph database schema mappings based on regular path queries, we prove that LAV simplification is PSPACEcomplete under the sound semantics, and in ExPSPACE in the case of exact schema mappings. By exploiting a language-theoretic characterization for containment of regular path queries with inverse (called two-way regular path queries) provided in [22], we also extend the results to the case where queries in schema mappings are two-way regular path queries, as well as conjunctive two-way regular path queries, and unions of such queries.

Note that a regular path query returns the set of node pairs in the graph database connected by a path conforming to the query, and therefore can be seen as the regular language constituted by all the words labeling the paths denoted by the query. Indeed, the simplification problem addressed in this paper has a language theoretic interpretation in terms of language equations. In general, solving systems of equations of the form $e=e^{\prime}$, where $e$ and $e^{\prime}$ are regular expressions over an alphabet of constants and variables is undecidable, because it is easy to express the universality problem for Context Free Grammars in this way. In [13], the authors study linear equations of the form

$$
e_{0}+e_{1} \cdot x_{1}+\cdots+e_{n} \cdot x_{n}=e_{0}^{\prime}+e_{1}^{\prime} \cdot x_{1}+\cdots+e_{n}^{\prime} \cdot x_{n}
$$

where $e_{0}, e_{0}^{\prime}, \ldots, e_{n}, e_{n}^{\prime}$ are regular expressions, and they prove that solving these equations is ExPTIME-complete. In contrast, we prove the solvability of another class of problems, namely, systems of language constraints of the form $e_{1} \sqsubseteq e_{2}$ and $e_{1}=e_{2}$ where $e_{2}$ has no variables. The key idea of our approach is that we can prove that solutions of the above equations are closed under congruence, which enables us to represent languages as graphs over the finite-state automaton for $e_{2}$. Taking into account the language-theoretic view, our work has also connections with $[4,42]$, which also study language constraints of the forms $e_{1} \sqsubseteq e_{2}$, and $e_{1}=e_{2}$. However, in these works, $e_{1}$ is restricted to be a single word on both the source and the target alphabets.

The paper is organized as follows. In Section 2, we recall some preliminary notions. In Section 3, we formally define the problem of schema mapping simplification based on logical implication. In Section 4, we study the problem in the case where queries and views are conjunctive queries, and unions thereof. In Section 5 we illustrate the techniques for the case of RPQs over graph databases, and in Section 6 we extend them to two-way RPQs, and to (unions of) conjunctive two-way RPQs. Section 7 briefly discusses GAV simplification, and Section 8 concludes the paper.

\section{PRELIMINARIES}

In this work we deal with two data models, the standard relational model [5], and the graph database model [21].

Given a (relational) alphabet $\Sigma$, a database $\mathcal{D}$ over $\Sigma$ is a finite structure over $\Sigma$. For a query $q$ over $\Sigma$, we denote with $q^{\mathcal{D}}$ the set of tuples resulting from evaluating $q$ in $\mathcal{D}$. A query $q$ over $\Sigma$ is empty if for each database $\mathcal{D}$ over $\Sigma$ we have $q^{\mathcal{D}}=\emptyset$. Given two queries $q_{1}$ and $q_{2}$ over $\Sigma$, we say that $q_{1}$ is contained in $q_{2}$, denoted $q_{1} \sqsubseteq q_{2}$, if $q_{1}^{\mathcal{D}} \subseteq q_{2}^{\mathcal{D}}$ for every database $\mathcal{D}$ over $\Sigma$. The queries $q_{1}$ and $q_{2}$ are equivalent, denoted $q_{1} \equiv q_{2}$, if both $q_{1} \sqsubseteq q_{2}$ and $q_{2} \sqsubseteq q_{1}$.

We assume familiarity with (unions of) conjunctive queries, (U)CQs, over a relational database. In particular, we consider such queries as interpreted under the active domain semantics [5]. The relational alphabets we are inter- ested in, always include two unary predicates true and false, returning respectively the active domain and the empty set. Hence, for every $n$, we can express what we call the universal query and the empty query of arity $n$, denoted true $/ n$, respectively false $/ n$, as the $\mathrm{CQ}$ that consist of one atom true $(x)$, respectively false $(x)$, for every distinguished variable $x$. We may omit $n$ when it is clear from the context. We recall that containment between (U)CQs can be characterized in terms of homomorphisms (also called containment mappings ) [24]: For two CQs $q_{1}$ and $q_{2}$, we have that $q_{1} \sqsubseteq q_{2}$ iff there is a homomorphisms from $q_{2}$ to $q_{1}$, i.e., a mapping $h$ from the variables and constants of $q_{2}$ to those of $q_{1}$ that is the identity on distinguished variables and constants and such that, if $r\left(x_{1}, \ldots, x_{k}\right)$ is an atom of $q_{2}$ with $r \neq$ true, then $r\left(h\left(x_{1}\right), \ldots, h\left(x_{k}\right)\right)$ is an atom of $q_{1}$. For two UCQ $q_{1}$ and $q_{2}$, we have that $q_{1} \sqsubseteq q_{2}$ iff for each CQ $q_{1}^{i}$ in $q_{1}$ there is a CQ $q_{2}^{j}$ in $q_{2}$ such that $q_{1}^{i} \sqsubseteq q_{2}^{j}$ [44].

We recall the basic notions regarding graph databases and regular path queries. A graph database is a finite graph whose nodes represent objects and whose edges are labeled by elements from an alphabet of binary relational symbols $[25,18,1,22]$. An edge $\left(o_{1}, r, o_{2}\right)$ from object $o_{1}$ to object $O_{2}$ labeled by $r$ represents the fact that relation $r$ holds between $o_{1}$ and $o_{2}$. A regular-path query (RPQ) over an alphabet $\Sigma$ of binary relation symbols is expressed as a regular expression or a nondeterministic finite state automaton (1NFA) over $\Sigma$. When evaluated on a graph database $\mathcal{D}$ over $\Sigma$, an RPQ $q$ computes the set $q^{\mathcal{D}}$ of pairs of objects connected in $\mathcal{D}$ by a path in the regular language $\mathcal{L}(q)$ defined by $q$.

We consider also two-way regular-path queries (2RPQs) [20, 22], which extend RPQs with the inverse operator. Formally, let $\Sigma^{ \pm}=\Sigma \cup\left\{r^{-} \mid r \in \Sigma\right\}$ be the alphabet including a new symbol $r^{-}$for each $r$ in $\Sigma$. Intuitively, $r^{-}$denotes the inverse of the binary relation $r$. If $p \in \Sigma^{ \pm}$, then we use $p^{-}$to mean the inverse of $p$, i.e., if $p$ is $r$, then $p^{-}$is $r^{-}$, and if $p$ is $r^{-}$, then $p^{-}$is $r$. 2RPQs are expressed by means of a 1 NFA over $\Sigma^{ \pm}$. When evaluated on a database $\mathcal{D}$ over $\Sigma$, a $2 \mathrm{RPQ} q$ computes the set $q^{\mathcal{D}}$ of pairs of objects connected in $\mathcal{D}$ by a semipath that conforms to the regular language $\mathcal{L}(q)$. A semipath in $\mathcal{D}$ from $x$ to $y$ (labeled with $p_{1} \cdots p_{n}$ ) is a sequence of the form $\left(y_{0}, p_{1}, y_{1}, \ldots, y_{n-1}, p_{n}, y_{n}\right)$, where $n \geq 0, y_{0}=x$, $y_{n}=y$, and for each $y_{i-1}, p_{i}, y_{i}$, we have that $p_{i} \in \Sigma^{ \pm}$, and, if $p_{i}=r$ then $\left(y_{i-1}, y_{i}\right) \in r^{\mathcal{D}}$, and if $p_{i}=r^{-}$then $\left(y_{i}, y_{i-1}\right) \in r^{\mathcal{D}}$. We say that a semipath $\left(y_{0}, p_{1}, \ldots, p_{n}, y_{n}\right)$ conforms to $q$ if $p_{1} \cdots p_{n} \in \mathcal{L}(q)$.

Finally, we consider conjunctions of 2RPQs and their unions, abbreviated (U)C2RPQs [19], which are (unions of) conjunctive queries constituted only by binary atoms whose predicate is a $2 \mathrm{RPQ}$. Specifically, a C2RPQ $q$ of arity $n$ is written in the form

$$
\left\{\left(x_{1}, \ldots, x_{n}\right) \mid q_{1}\left(y_{1}, y_{2}\right) \wedge \cdots \wedge q_{m}\left(y_{2 m-1}, y_{2 m}\right)\right\}
$$

where $x_{1}, \ldots, x_{n}, y_{1}, \ldots, y_{2 m}$ range over a set $\left\{z_{1}, \ldots, z_{k}\right\}$ of variables, $\left\{x_{1}, \ldots, x_{n}\right\} \subseteq\left\{y_{1}, \ldots, y_{2 m}\right\}$, and each $q_{j}$ is a $2 \mathrm{RPQ}$. When evaluated over a database $\mathcal{D}$ over $\Sigma$, the C2RPQ $q$ computes the set of tuples $\left(o_{1}, \ldots, o_{n}\right)$ of objects such that there is a total mapping $\varphi$ from $\left\{z_{1}, \ldots, z_{k}\right\}$ to the objects in $\mathcal{D}$ with $\varphi\left(x_{i}\right)=o_{i}$, for $i \in\{1, \ldots, n\}$, and $\left(\varphi\left(y_{2 j-1}\right), \varphi\left(y_{2 j}\right)\right) \in q_{j}^{\mathcal{D}}$, for $j \in\{1, \ldots, m\}$.

We conclude by observing that (U)CQs, RPQs, 2RPQs, and $(\mathrm{U}) \mathrm{C} 2 \mathrm{RPQs}$ are monotone, where a query $q$ is monotone 
if, whenever $\mathcal{D}_{1} \subseteq \mathcal{D}_{2}$ (i.e., $r^{\mathcal{D}_{1}} \subseteq r^{\mathcal{D}_{2}}$ for each relation $r$ ) we have that $q^{\mathcal{D}_{1}} \subseteq q^{\mathcal{D}_{2}}$.

\section{SCHEMA MAPPING SIMPLIFICATION}

We refer to a scenario with one source schema, one target schema, and a schema mapping between the two. To model the source and the target schemas we refer to two finite alphabets, the source alphabet $\Sigma_{s}$ and the target alphabet $\Sigma_{t}$, and to specify the mapping, we use a correspondence between queries expressed in a given query language.

Definition 1. Given a query language $\mathcal{Q}$, a $\mathcal{Q}$-based (schema) mapping assertion from $\Sigma_{s}$ to $\Sigma_{t}$ is a statement of the form $q_{s} \leadsto q_{t}$, where $q_{s}$ and $q_{t}$ are two queries in $\mathcal{Q}$ with the same arity, respectively over $\Sigma_{s}$ and over $\Sigma_{t} . A \mathcal{Q}$ based (schema) mapping from $\Sigma_{s}$ to $\Sigma_{t}$ is a set of mapping assertions from $\Sigma_{s}$ to $\Sigma_{t}$.

In the following, we specify explicitly $\mathcal{Q}, \Sigma_{s}$, and $\Sigma_{t}$ only when they are required or not clear from the context.

We consider two types of schema mappings, called sound and exact. Intuitively, in a sound mapping, the correspondence between the tuples computed by $q_{s}$ and those computed by $q_{t}$ is set containment, while in an exact mapping the correspondence is set equality. Formally, given a source database $\mathcal{D}_{s}$ and a target database $\mathcal{D}_{t}$, we say that a sound mapping $M$ is satisfied by $\left(\mathcal{D}_{s}, \mathcal{D}_{t}\right)$, denoted $\left(\mathcal{D}_{s}, \mathcal{D}_{t}\right) \models M$, if for each mapping assertion $q_{s} \leadsto q_{t}$ in $M$, we have that $q_{s}^{\mathcal{D}_{s}} \subseteq q_{t}^{\mathcal{D}_{t}}$. Similarly, an exact mapping $M$ is satisfied by $\left(\mathcal{D}_{s}, \overline{\mathcal{D}}_{t}\right)$ if for each $q_{s} \leadsto q_{t}$ in $M$, we have that $q_{s}^{\mathcal{D}_{s}}=q_{t}^{\mathcal{D}_{t}}$.

A fundamental notion in our setting is that of logical implication between mappings.

Definition 2. A mapping $M_{1}$ logically implies a mapping $M_{2}$, denoted $M_{1} \models M_{2}$, if for every pair $\left(\mathcal{D}_{s}, \mathcal{D}_{t}\right)$ such that $\left(\mathcal{D}_{s}, \mathcal{D}_{t}\right) \mid=M_{1}$, we also have that $\left(\mathcal{D}_{s}, \mathcal{D}_{t}\right) \models M_{2}$.

We consider two simplified forms of mappings called LAV (local-as-view) and GAV (global-as-view), respectively. A LAV assertion is an assertion $q_{s} \leadsto q_{t}$, where $q_{s}$ is constituted simply by an atom whose predicate symbol belongs to $\Sigma_{s}$, while $q_{t}$ is an arbitrary query ${ }^{1}$. Conversely, in a GAV assertion, $q_{t}$ is constituted simply by an atom (whose predicate symbol belongs to $\Sigma_{t}$ ), while $q_{s}$ is an arbitrary query. A LAV mapping is a set of LAV assertions with one assertion for each symbol in $\Sigma_{s}$. If $M_{L}$ is a LAV mapping and $a \in \Sigma_{s}$, we denote with $M_{L}(a)$ the target query to which $a$ is mapped by $M_{L}$. Conversely, a GAV mapping is a set of GAV assertions with one assertion for each symbol in $\Sigma_{t}$. Analogously to the case of LAV mappings, $M_{G}(a)$ denotes the source query to which the symbol $a \in \Sigma_{t}$ is mapped by the GAV mapping $M_{G}$. Note that some of the queries in a LAV (resp., GAV) mapping may be the empty query.

The problem we consider aims at checking whether a simplified mapping exists that logically implies a given mapping $M$. We would like to rule out simplified mappings that trivially imply $M$ by making the query on the left-hand (resp., right-hand) side of some mapping assertion of $M$ evaluate to $\emptyset$ (resp., the active domain).

${ }^{1}$ For RPQs and 2RPQs, where query variables are not represented explicitly, we consider an atom to be simply a binary predicate symbol.
Definition 3. A LAV (resp., GAV) mapping $M^{\prime}$ is said to trivially imply a mapping $M$, denoted $M^{\prime} \models_{\text {triv }} M$, if there is a mapping assertion $q_{s} \leadsto q_{t} \in M$ such that for each pair $\left(\mathcal{D}_{s}, \mathcal{D}_{t}\right)$ with $\left(\mathcal{D}_{s}, \mathcal{D}_{t}\right) \models M^{\prime}$, we have that $q_{s}^{\mathcal{D}_{s}}=$ false $^{\mathcal{D}_{s}}$ (resp., $q_{t}^{\mathcal{D}_{t}}=$ true $^{\mathcal{D}_{t}}$ ).

DEFINITION 4. Let $t$ be one of SOUND or EXACT, $f$ one of $\mathrm{LAV}$ or $\mathrm{GAV}$, and $\mathcal{Q}_{1}$ and $\mathcal{Q}_{2}$ two query languages. Mapping simplification, denoted

$$
\operatorname{MSimp}\left[t, f, \mathcal{Q}_{1}, \mathcal{Q}_{2}\right],
$$

is the following decision problem: given a $\mathcal{Q}_{1}$-based schema mapping $M$ of type $t$, check whether there exists a $\mathcal{Q}_{2}$-based schema mapping $M^{\prime}$ of type $t$ and form $f$ such that $M^{\prime} \models M$, and $M^{\prime} \forall_{\text {triv }} M$.

If a simplified mapping exists, we are also interested in actually computing one. Therefore, we consider the corresponding synthesis problem.

DEFINITION 5. Let $t$ be one of SOUND or EXACT, $f$ one of $\mathrm{LAV}$ or $\mathrm{GAV}$, and $\mathcal{Q}_{1}$ and $\mathcal{Q}_{2}$ two query languages. Mapping synthesis, denoted,

$\operatorname{MSYNT}\left[t, f, \mathcal{Q}_{1}, \mathcal{Q}_{2}\right]$,

is the following problem: given a $\mathcal{Q}_{1}$-based schema mapping $M$ of type $t$, find a $\mathcal{Q}_{2}$-based schema mapping $M^{\prime}$ of type $t$ and form $f$ such that $M^{\prime}=M$, and $M^{\prime} \not \forall_{\text {triv }} M$.

To rule out (uninteresting) cases where all LAV (or GAV) mappings that imply a given mapping $M$ do so trivially, in the following we require that for each mapping assertion $q_{s} \sim q_{t} \in M$, both $q_{s}$ and $q_{t}$ are different from false.

In general we are interested in the tightest simplification of a mapping $M$, i.e., the simplification that better approximates $M$. Hence, we also consider the maximal mapping synthesis problem, MAxMSynt $\left[t, f, \mathcal{Q}_{1}, \mathcal{Q}_{2}\right]$, where, given a $\mathcal{Q}_{1}$-based mapping $M$ of type $t$, we aim at computing a $\mathcal{Q}_{2}-$ based mapping $M^{\prime}$ of type $t$ and form $f$ such that $M^{\prime}=M$ and there is no $\mathcal{Q}_{2}$-based mapping $M^{\prime \prime}$ of type $t$ and form $f$ such that $M^{\prime \prime} \models M, M^{\prime} \models M^{\prime \prime}$, and $M^{\prime \prime} \not M^{\prime}$.

In this paper we study the above problems for a variety of cases, where $\mathcal{Q}_{1}$ and $\mathcal{Q}_{2}$ range over (U)CQs and variants of queries over graph databases.

We start by observing that we can characterize mapping implication, and hence mapping simplification, in terms of query unfolding wrt a set of mappings. We make use of such a characterization in the technical development in the subsequent sections. The notion of query unfolding is formally defined as follows: let $q_{s}$ be a source query and $M_{L}$ a LAV mapping. The unfolding of $q_{s}$ wrt $M_{L}$, denoted $q_{s}\left[M_{L}\right]$, is the target query obtained by replacing each atom $\alpha$ in $q_{s}$ whose predicate symbol is $a$ with $M_{L}(a)$. An analogous definition holds for the unfolding $q_{t}\left[M_{G}\right]$ of a target query $q_{t}$ wrt a GAV mapping $M_{G}$.

TheOREM 6. Let $\mathcal{Q}$ be a monotone query language.

(1) Let $M_{L}$ be a LAV mapping and $M$ a mapping, both $\mathcal{Q}$ based and of type SOUND (resp., EXACT). Then $M_{L}=M$ iff for each assertion $q_{s} \leadsto q_{t}$ in $M$, we have that $q_{s}\left[M_{L}\right] \sqsubseteq q_{t}$ (resp., $q_{s}\left[M_{L}\right] \equiv q_{t}$ ).

(2) Let $M_{G}$ be a GAV mapping and $M$ a mapping, both $\mathcal{Q}$ based and of type SOUND (resp., EXACT). Then $M_{G}=M$ iff for each assertion $q_{s} \leadsto q_{t}$ in $M$, we have that $q_{s} \sqsubseteq q_{t}\left[M_{G}\right]$ (resp., $q_{s} \equiv q_{t}\left[M_{G}\right]$ ). 
Proof (SKETCH). We provide the proof for (1), in particular for the case of sound mappings. The other cases can be proved analogously.

We start with the following observation, which is easy to prove: if $M_{L}$ is a LAV mapping, and $\mathcal{D}_{s}$ is the source database obtained from a target database $\mathcal{D}_{t}$ by letting $r^{\mathcal{D}_{s}}=M_{L}(r)^{\mathcal{D}_{t}}$ for every $r \in \Sigma_{s}$, then for every source query $q$, we have that $q^{\mathcal{D}_{s}}=q\left[M_{L}\right]^{\mathcal{D}_{t}}$.

" $\Rightarrow$ " Now, assume that there is an assertion $q_{s} \leadsto q_{t}$ in $M$ such that $q_{s}\left[M_{L}\right] \nsubseteq q_{t}$, and let $\mathcal{D}_{t}$ be such that for some tuple $\mathbf{d}$ we have $\mathbf{d} \in q_{s}\left[M_{L}\right]^{\mathcal{D}_{t}}$, and $\mathbf{d} \notin q_{t}^{\mathcal{D}_{t}}$. Let $\mathcal{D}_{s}$ be the source database obtained from $\mathcal{D}_{t}$ by letting $r^{\mathcal{D}_{s}}=M_{L}(r)^{\mathcal{D}_{t}}$ for every $r \in \Sigma_{s}$. Clearly, $\left(\mathcal{D}_{s}, \mathcal{D}_{t}\right) \models M_{L}$. By the above observation, we have that $q_{s}^{\mathcal{D}_{s}}=q_{s}\left[M_{L}\right]^{\mathcal{D}_{t}}$, and therefore $\mathbf{d} \in q_{s}^{\mathcal{D}_{s}}$. It follows that $\left(\mathcal{D}_{s}, \mathcal{D}_{t}\right) \not \models q_{s} \leadsto q_{t}$, and $M_{L} \not \forall M$. " $\Leftarrow$ " Assume that $M_{L} \not \models M$, i.e., there is an assertion $q_{s} \leadsto q_{t}$ in $M$, and a pair $\left(\mathcal{D}_{s}, \mathcal{D}_{t}\right)$ such that $\left(\mathcal{D}_{s}, \mathcal{D}_{t}\right) \models$ $M_{L}$, and $q_{s}^{\mathcal{D}_{s}} \nsubseteq q_{t}^{\mathcal{D}_{t}}$, which means that there is $\mathbf{d}$ such that $\mathbf{d} \in q_{s}^{\mathcal{D}_{s}}$ but $\mathbf{d} \notin q_{t}^{\mathcal{D}_{t}}$. Let $\mathcal{D}_{s}^{\prime}$ be such that $r^{\mathcal{D}_{s}^{\prime}}=M_{L}(r)^{\mathcal{D}_{t}}$ for every $r \in \Sigma_{s}$. Clearly, $\mathcal{D}_{s}^{\prime} \supseteq \mathcal{D}_{s}$, and $\left(\mathcal{D}_{s}^{\prime}, \mathcal{D}_{t}\right) \models M_{L}$. Since $q_{s}$ is monotone, we have that $\mathbf{d} \in q_{s}^{\mathcal{D}_{s}^{\prime}}=q_{s}\left[M_{L}\right]^{\mathcal{D}_{t}}$, and therefore $q_{s}\left[M_{L}\right] \nsubseteq q_{t}$.

THEOREM 7. Let $M$ be a mapping and $M^{\prime}$ a LAV (resp., GAV) mapping. Then $M^{\prime} \models_{\text {triv }} M$ iff for some mapping assertion $q_{s} \leadsto q_{t} \in M$ we have that $q_{s}\left[M^{\prime}\right] \sqsubseteq$ false (resp., true $\left.\sqsubseteq q_{t}\left[M^{\prime}\right]\right)$.

Proof Sketch. Similar to that of Theorem 6.

For many of the results in the next sections, we make use of the above characterization, without further mentioning Theorems 6 and 7 .

\section{LAV SIMPLIFICATION FOR (U)CQs}

In this section, we consider the case of mappings based on conjunctive queries (CQs) and their unions (UCQs), and study the problem of simplifying a given mapping $M$ in terms of a LAV mapping. The techniques we adopt for establishing our upper bounds are based on determining a polynomial bound on the length of the queries to consider when searching for the LAV mapping logically implying $M$, and are reminiscent of those in [39].

In the following, when we refer to a LAV mapping logically implying a given mapping, we implicitly assume that implication is non-trivial. We start with the problem of simplifying a CQ-based mapping in terms of a CQ-based LAV mapping.

TheOREM 8. Both MSimp[SOUND,LAV,CQ,CQ] and MSIMP[EXACT, LAV, CQ, CQ] are in NP.

Proof. Consider a sound CQ-based mapping consisting of a single assertion $q_{s} \leadsto q_{t}$, where $q_{t}$ contains $\ell_{q_{t}}$ atoms, and a sound CQ-based LAV mapping $M_{L}$ such that $q_{s}\left[M_{L}\right] \sqsubseteq$ $q_{t}$. Then, there exists a homomorphism from $q_{t}$ to $q_{s}\left[M_{L}\right]$, and at most $\ell_{q_{t}}$ atoms of $q_{s}\left[M_{L}\right]$ are in the image of this homomorphism. Hence, for each symbol $a \in \Sigma_{s}$ occurring in $q_{s}$, only at most $\ell_{q_{t}}$ atoms in query $M_{L}(a)$ are needed for the homomorphism. In the general case where the mapping $M$ consists of several assertions, for each $a \in \Sigma_{s}$ we need at most $\ell_{M}=\sum_{q_{s} \sim q_{t} \in M} \ell_{q_{t}}$ atoms in the query $M_{L}(a)$, in order to guarantee the existence of the homomorphisms for all the assertions in $M$. Hence, in order to check for the existence of an appropriate LAV mapping $M_{L}$, it suffices to guess, for each symbol $a \in \Sigma_{s}$ appearing in one of the mapping assertions in $M$, a CQ $M_{L}(a)$ over $\Sigma_{t}$ of size at most $\ell_{M}$, and check that $q_{s}\left[M_{L}\right] \sqsubseteq q_{t}$, for each $q_{s} \leadsto q_{t} \in M$. In doing so, we rule out the guess of mappings that trivially imply $M$. This gives us immediately an NP upper bound for MSimP[SOUND,LAV,CQ,CQ].

For MSimp[EXACT,LAV,CQ,CQ], in addition to checking that $q_{s}\left[M_{L}\right] \sqsubseteq q_{t}$, we need also to check that $q_{t} \sqsubseteq q_{s}\left[M_{L}\right]$. We observe that the bound on the number of atoms in $M_{L}(a)$ derived for the sound case is still valid, since if $q_{t} \sqsubseteq q_{s}\left[M_{L}\right]$ for a LAV mapping $M_{L}$, then also $q_{t} \sqsubseteq q_{s}\left[M_{L}^{\prime}\right]$ for every LAV mapping $M_{L}^{\prime}$ such that $M_{L}^{\prime}(a)$ is constituted by a subset of the atoms of $M_{L}(a)$. Therefore, the overall complexity does not change.

For the case where $M$ is UCQ-based, and the LAV mapping $M_{L}$ is still CQ-based, we can generalize the above argument by considering containment between UCQs instead of containment between CQs.

Theorem 9. Both MSimp[SOUnd,LAV,UCQ,CQ] and MSimP [EXACT,LAV, UCQ,CQ] are in NP.

The last case we consider is the one where both $M$ and the LAV mapping $M_{L}$ are UCQ-based. In the sound case, we show that simplification to a UCQ-based LAV mapping is equivalent to simplification to a CQ-based LAV mapping.

LEMMA 10. MSimP[SOUND,LAV,UCQ,UCQ] admits a solution for a mapping $M$ iff MSIMP[SOUND,LAV, UCQ,CQ] admits a solution for $M$.

Proof. Let $M$ be a sound UCQ-based mapping and $M_{L}$ a sound UCQ-based LAV mapping such that $M_{L} \models M$. Consider the CQ-based LAV mapping $M_{L}^{\prime}$ obtained from $M_{L}$ by choosing, for each symbol $a$ in $\Sigma_{s}$, as $M_{L}^{\prime}(a)$ one of the CQs in $M_{L}(a)$. We show that $M_{L}^{\prime} \forall \forall_{\text {triv }} M$, and that $M_{L}^{\prime} \models M$. Consider one assertion $q_{s} \leadsto q_{t} \in M$ such that $q_{s}\left[M_{L}\right]$ is a non-empty positive query. Such a mapping assertion exists, since $M_{L} \not \models_{\text {triv }} M$. Then, $q_{s}\left[M_{L}^{\prime}\right]$ is a non-empty UCQ, and hence $M_{L}^{\prime} \not \nvdash_{\text {triv }} M$. To show that $M_{L}^{\prime}=M$, it is sufficient to observe that, for each assertion $q_{s} \leadsto q_{t} \in M$, each CQ in $q_{s}\left[M_{L}^{\prime}\right]$ is contained in $q_{s}\left[M_{L}\right]$, and hence in $q_{t}$.

By the above lemma, we trivially get:

Theorem 11. MSimp[SOUND, LAV, UCQ,UCQ] is in NP.

In the exact case, if we allow for UCQ-based LAV mappings, we get a higher upper-bound.

Theorem 12. MSimp[EXACT,LAV,UCQ,UCQ] is in $\Pi_{2}^{p}$.

We now show that the upper bounds for the sound cases established in Theorems 8, 9, and 11 are tight.

Theorem 13. MSimp[SOUnd, LAV,CQ,CQ] is NP-hard.

Proof. The proof is by a reduction from 3-colorability. Consider a graph $G=(N, E)$, with $N=\left\{n_{1}, \ldots, n_{k}\right\}$.

Let $\Sigma_{s}=\left\{t / 2, a_{s} / 2, a_{f} / 2\right\}, \Sigma_{t}=\left\{e / 2, b_{s} / 2, b_{f} / 2\right\}$,

$$
\begin{aligned}
q_{T}=\{(s, f) \mid & a_{s}(s, r), a_{s}(s, g), a_{s}(s, b), \\
& t(r, g), t(g, r), t(r, b), t(b, r), t(g, b) \\
& \left.a_{f}(r, f), a_{f}(g, f), a_{f}(b, f)\right\} \\
q_{G}=\{(s, f) \mid & b_{s}\left(s, x_{1}\right), \ldots, b_{s}\left(s, x_{k}\right), \\
& \bigwedge_{\left(n_{i}, n_{j}\right) \in E}\left\{e\left(x_{i}, x_{j}\right), e\left(x_{j}, x_{i}\right)\right\}, \\
& \left.b_{f}\left(x_{1}, f\right), \ldots, b_{f}\left(x_{k}, f\right)\right\}
\end{aligned}
$$$$
t(r, g), t(g, r), t(r, b), t(b, r), t(g, b), t(b, g) \text {, }
$$ 
and define the following mapping $M$ :

$$
\begin{aligned}
q_{T} & \leadsto q_{G} \\
\{(x, y) \mid t(x, y)\} & \sim\{(x, y) \mid e(x, y)\} \\
\left\{(x, y) \mid a_{s}(x, y)\right\} & \sim\left\{(x, y) \mid b_{s}(x, y)\right\} \\
\left\{(x, y) \mid a_{f}(x, y)\right\} & \leadsto\left\{(x, y) \mid b_{f}(x, y)\right\}
\end{aligned}
$$

Intuitively, assertion (1) maps a triangle, whose three vertexes are connected by $a_{s}$ and $a_{f}$ to the distinguished variables $s$ and $f$ respectively, to the graph $G$, whose nodes are connected by $b_{s}$ and $b_{f}$ to the distinguished variables $s$ and $f$ respectively.

One can show that $G$ is 3-colorable iff MSimp[SOUND,LAV,CQ,CQ] with input $M$ admits a solution.

Corollary 14. MSimp[SOUnd,LAV,UCQ,CQ] and MSIMP[SOUND,LAV,UCQ,UCQ] are NP-hard.

Proof. From Theorem 13 we trivially get the result for MSimP[SOUND,LAV,UCQ,CQ], and by considering Lemma 10, we get the result also for MSIMP[SOUND,LAV,UCQ,UCQ].

We conjecture that the upper bounds we provided for the case where schema mappings are of type exact are tight.

\section{LAV SIMPLIFICATION FOR RPQs}

In this section, we consider the case of RPQs over graph databases, and study the problem of simplifying an RPQbased mapping in terms of an RPQ-based LAV mapping. For our results, we exploit a straightforward language theoretic characterization of containment between RPQs.

TheOrem 15 ([21]). Let $q_{1}, q_{2}$ be two RPQs, and $\mathcal{L}\left(q_{1}\right), \mathcal{L}\left(q_{2}\right)$ the corresponding regular languages. Then $q_{1} \sqsubseteq q_{2}$ iff $\mathcal{L}\left(q_{1}\right) \subseteq \mathcal{L}\left(q_{2}\right)$.

In the following, we identify an RPQ $q$ over an alphabet $\Sigma$ with the language over $\Sigma$ accepted by the regular expression (RE) or 1NFA representing $q$. Considering the languagetheoretic characterization above, it follows from Theorems 6 and 7 that, if $M_{L}$ is a LAV mapping and $M$ a mapping, both of type sOUND (resp., EXACT), then $M_{L} \models M$ and $M_{L} \forall_{\text {triv }} M$ iff for each assertion $q_{s} \leadsto q_{t}$ in $M$, we have that $q_{s}\left[M_{L}\right] \subseteq q_{t}$ (resp., $\left.q_{s}\left[M_{L}\right]=q_{t}\right)$ and $q_{s}\left[M_{L}\right] \neq \emptyset$. Here, the unfolding $q_{s}\left[M_{L}\right]$ of $q_{s}$ wrt $M_{L}$ denotes the language over $\Sigma_{t}$ obtained from $q_{s}$ by expanding in each word in $q_{s}$ each symbol $a \in \Sigma_{s}$ with the language $M_{L}(a)$.

We start by showing that we can characterize mapping implication $M_{L}=M$ between a LAV mapping $M_{L}$ and a mapping $M$ in terms of a single language containment (for sound mappings) or language equality (for exact mappings). For this, we extend the notion of unfolding of $q_{s}$ wrt $M_{L}$ to the case where $q_{s}$ may contain additional symbols wrt those in $\Sigma_{s}$. In particular, the additional symbols are left unchanged by the unfolding.

Proposition 16. Let $M$ be an RPQ-based mapping of type SOUND (resp., EXACT) from $\Sigma_{s}$ to $\Sigma_{t}$, and let \# be a symbol not in $\Sigma_{s} \cup \Sigma_{t}$. Then there are RPQs $q_{M, s}$ over $\Sigma_{s} \cup$ $\{\#\}$ and $q_{M, t}$ over $\Sigma_{t} \cup\{\#\}$, both of size linear in $M$, such that an RPQ-based LAV mapping $M_{L}$ of type SOUND (resp., EXACT) is a solution to MSYNT[SOUND,LAV,RPQ,RPQ] (resp., MSYNT[EXACT,LAV,RPQ,RPQ]) with input $M$ iff $q_{M, s}\left[M_{L}\right] \subseteq$ $q_{M, t}\left(\right.$ resp., $\left.q_{M, s}\left[M_{L}\right]=q_{M, t}\right)$ and $q_{M, s}\left[M_{L}\right] \neq \emptyset$.
Proof. Let $M=\left\{q_{1, s} \leadsto q_{1, t}, \ldots, q_{k, s} \leadsto q_{k, t}\right\}$. We set $q_{M, s}=q_{1, s} \cdot \# \cdots \# \cdot q_{k, s}$ and $q_{M, t}=q_{1, t} \cdot \# \cdots \# \cdot q_{k, t}$. Intuitively, the fresh symbol \# acts as a separator for the different parts of $q_{M, s}$ and $q_{M, t}$. It is easy to verify that, for every LAV mapping $M_{L}$, we have that $q_{i, s}\left[M_{L}\right] \subseteq q_{i, t}$ (resp., $\left.q_{i, s}\left[M_{L}\right]=q_{i, t}\right)$ for $i \in\{1, \ldots, k\}$ iff $q_{M, s}\left[M_{L}\right] \subseteq q_{M, t}$ (resp., $\left.q_{M, s}\left[M_{L}\right]=q_{M, t}\right)$, and that $q_{i, s}\left[M_{L}\right] \neq \emptyset$ for $i \in\{1, \ldots, k\}$ iff $q_{M, s}\left[M_{L}\right] \neq \emptyset$.

In the following, let $\Sigma_{n}$ be an alphabet of new symbols disjoint from $\Sigma_{s}$ and $\Sigma_{t}$, and let $\Sigma_{s}^{\prime}=$ $\Sigma_{s} \cup \Sigma_{n}$ and $\Sigma_{t}^{\prime}=\Sigma_{t} \cup \Sigma_{n}$. By Proposition 16, the problem MSimP[SOUND,LAV,RPQ,RPQ] (or MSIMP[EXACT,LAV,RPQ,RPQ]) can be polynomially reduced to the problem of checking, whether for languages $q_{s}$ over $\Sigma_{s}^{\prime}$ and $q_{t}$ over $\Sigma_{t}^{\prime}$ there is a LAV mapping $M_{L}$ such that $q_{s}\left[M_{L}\right] \subseteq q_{t}$ (resp., $\left.q_{s}\left[M_{L}\right]=q_{t}\right)$ and $q_{s}\left[M_{L}\right] \neq \emptyset$.

Our technique for mapping simplification exploits a characterization of regular languages by means of congruence classes $[43,23,42]$. Let $A_{t}=\left(\Sigma_{t}^{\prime}, S_{t}, s_{t}^{0}, \delta_{t}, F_{t}\right)$ be a $1 \mathrm{NFA}$ for $q_{t}$. Then $A_{t}$ defines a set of congruence classes partitioning $\Sigma_{t}^{\prime *}$. Each congruence class is characterized by a binary relation $G \subseteq S_{t} \times S_{t}$ (i.e., a directed graph over $S_{t}$ ), and we define the congruence class associated with $G$ as

$$
\begin{aligned}
& \mathcal{L}(G)=\left\{w \in \Sigma_{t}^{*} \mid \text { for all } s_{1}, s_{2} \in S_{t},\right. \\
& \left.s_{2} \in \delta_{t}\left(s_{1}, w\right) \text { iff }\left(s_{1}, s_{2}\right) \in G\right\} .
\end{aligned}
$$

Intuitively, each word $w \in \mathcal{L}(G)$ connects $s_{1}$ to $s_{2}$ in $A_{t}$, for each pair $\left(s_{1}, s_{2}\right) \in G$. For a word $w \in \Sigma_{t}^{\prime *}$, we denote with $[w]_{A_{t}}$ the congruence class to which $w$ belongs.

It follows immediately from the characterization of congruence classes in terms of binary relations over the states of $A_{t}$ that the set of congruence classes is closed under concatenation. Specifically, for two congruence classes $\mathcal{L}\left(G_{1}\right)$ and $\mathcal{L}\left(G_{2}\right)$, respectively with associated relations $G_{1}$ and $G_{2}$, the binary relation associated with $\mathcal{L}\left(G_{1}\right) \cdot \mathcal{L}\left(G_{2}\right)$ is $G_{1} \circ G_{2}{ }^{2}$

We introduce some notation that we use here, and later in this section:

- $\mathcal{G}_{t}=2^{S_{t} \times S_{t}}$ denotes the set of binary relations associated with the congruence classes for $A_{t}$,

- $G_{t}^{\varepsilon}=\left\{(s, s) \mid s \in S_{t}\right\}$, and

- $G_{t}^{b}=\left\{\left(s_{1}, s_{2}\right) \mid s_{2} \in \delta_{t}\left(s_{1}, b\right)\right\}$, for each $b \in \Sigma_{t}^{\prime}$.

Then, for each $G \in \mathcal{G}_{t}$, we can characterize the congruence class $\mathcal{L}(G)$ associated with $G$ in terms of a DFA.

Lemma 17 ([43]). The language $\mathcal{L}(G)$ is accepted by the DFA $A_{G}=\left(\Sigma_{t}^{\prime}, \mathcal{G}_{t}, G_{t}^{\varepsilon}, \delta_{A_{t}}, F_{G}\right)$, where $F_{G}=\{G\}$ and $\delta_{A_{t}}(R, b)=R \circ G_{t}^{b}$, for each $R \subseteq S_{t} \times S_{t}$ and $b \in \Sigma_{t}^{\prime}$.

Notice that, if $A_{t}$ has $m$ states, then $A_{G}$ has $2^{m^{2}}$ states.

We observe next that we need to allow for the presence of empty queries in the LAV mapping we are looking for. Consider, e.g., $q_{s}=\left(a_{1}+a_{3}\right) \cdot\left(a_{2}+a_{3}\right)$ and $q_{t}=b_{1} \cdot b_{2}$. It is easy to see that for all LAV mappings $M_{L}$ such that $q_{s}\left[M_{L}\right] \subseteq q_{t}$, we have that $M_{L}\left(a_{3}\right)=\emptyset$. One such LAV mapping $M_{L}$ is

$$
M_{L}\left(a_{1}\right)=b_{1}, \quad M_{L}\left(a_{2}\right)=b_{2}, \quad M_{L}\left(a_{3}\right)=\emptyset .
$$

Observe also that $\left[b_{1}\right]_{A_{t}}=\left\{b_{1}\right\}$ and $\left[b_{2}\right]_{A_{t}}=\left\{b_{2}\right\}$, where $A_{t}$ is the obvious 1NFA for $b_{1} \cdot b_{2}$.

${ }^{2}$ We use $L_{1} \cdot L_{2}$ to denote concatenation between languages, and $G_{1} \circ G_{2}$ to denote composition of binary relations. 


\subsection{Upper bounds for sound mappings}

We first deal with the case of sound mappings, and prove two preliminary results. The first lemma states that w.l.o.g. we can restrict the attention to LAV mappings in which the queries are singletons, i.e., queries that are either empty or constituted by a single word.

LEMMA 18. Let $q_{s}$ be an $R P Q$ over $\Sigma_{s}^{\prime}$, and $q_{t}$ an $R P Q$ over $\Sigma_{t}^{\prime}$. If there exists an RPQ-based LAV mapping $M_{L}$ such that $q_{s}\left[M_{L}\right] \subseteq q_{t}$, then there exists an RPQ-based LAV mapping $M_{L}^{\prime}$ such that $q_{s}\left[M_{L}^{\prime}\right] \subseteq q_{t}$ in which each query is either a single word over $\Sigma_{t}$ or empty.

The next lemma shows that one can close queries in LAV mappings under congruence.

LEMMA 19. Let $q_{s}$ be an $R P Q$ over $\Sigma_{s}^{\prime}, q_{t}$ an $R P Q$ over $\Sigma_{t}^{\prime}$ expressed through a $1 N F A A_{t}$, and $M_{L}$ a singleton mapping such that $q_{s}\left[M_{L}\right] \subseteq q_{t}$. Then, for $M_{L}^{\prime}$ defined such that

$$
M_{L}^{\prime}(a)= \begin{cases}{\left[w^{a}\right]_{A_{t}},} & \text { if } M_{L}(a)=w^{a} \\ \emptyset, & \text { if } M_{L}(a)=\emptyset,\end{cases}
$$

we have that $q_{s}\left[M_{L}^{\prime}\right] \subseteq q_{t}$.

From these two lemmas we get that, when searching for a LAV mapping $M_{L}$ satisfying $q_{s}\left[M_{L}\right] \subseteq q_{t}$, we can restrict the attention to queries that are congruence classes for $A_{t}$.

LEMMA 20. Let $q_{s}$ be an $R P Q$ over $\Sigma_{s}^{\prime}$, and $q_{t}$ an $R P Q$ over $\Sigma_{t}^{\prime}$ expressed through a $1 N F A A_{t}$. If there exists an RPQ-based LAV mapping $M_{L}$ such that $q_{s}\left[M_{L}\right] \subseteq q_{t}$, then there exists an RPQ-based LAV mapping $M_{L}^{\prime}$ such that $q_{s}\left[M_{L}^{\prime}\right] \subseteq q_{t}$, and such that $M_{L}^{\prime}(a)$ is a congruence class for $A_{t}$, for each $a \in \Sigma_{s}$.

PROOF. If there exist an RPQ-based LAV mapping $M_{L}$ such that $q_{s}\left[M_{L}\right] \subseteq q_{t}$, then by Lemma 18, w.l.o.g., we can assume that $M_{L}$ consists of singleton queries. Then, the claim follows from Lemma 19.

We derive now a procedure that, given an RPQ $q_{s}$ over $\Sigma_{s}^{\prime}$, and an RPQ $q_{t}$ over $\Sigma_{t}^{\prime}$ expressed respectively through $1 \mathrm{NFAs} A_{s}=\left(\Sigma_{s}^{\prime}, S_{s}, s_{s}^{0}, \delta_{s}, F_{s}\right)$ and $A_{t}=\left(\Sigma_{t}^{\prime}, S_{t}, s_{t}^{0}, \delta_{t}, F_{t}\right)$, checks for the existence of a sound RPQ-based LAV mapping $M_{L}$ such that

(1) $q_{s}\left[M_{L}\right] \neq \emptyset$, and

(2) $q_{s}\left[M_{L}\right] \subseteq q_{t}$

Specifically, by Lemma 20, it is sufficient to consider LAV mappings in which each query is constituted by a single congruence class, which can be represented by a binary relation over the state set $S_{t}$ of $A_{t}$. Hence, for each $a \in \Sigma_{s}$, we guess such a binary relation $G_{a}$ and verify that for the LAV mapping $M_{L}$ defined by $M_{L}(a)=\mathcal{L}\left(G_{a}\right)$, conditions (1) and (2) are satisfied. In doing so, we exploit Lemma 17, which provides a characterization of $\mathcal{L}\left(G_{a}\right)$ in terms of a DFA $A_{G_{a}}$.

To check condition (1), we proceed as follows:

1 . for each $a \in \Sigma_{s}$, we check whether $a$ is a bad symbol, i.e., whether $\mathcal{L}\left(G_{a}\right)=\emptyset$;

2. we delete from $A_{s}$ each transition labeled by a bad symbol; and

3. we check whether the resulting 1NFA accepts a nonempty language.

To check condition (2), we proceed as follows:
1. we construct an $1 \mathrm{NFA} A_{M_{L}}$ accepting $q_{s}\left[M_{L}\right]$;

2. we construct the $1 \mathrm{NFA} A_{\nsubseteq}=A_{M_{L}} \times \bar{A}_{t}$ as the product NFA of $A_{M_{L}}$ and the 1NFA $\bar{A}_{t}$ accepting $\Sigma_{t}^{\prime *} \backslash q_{t}$;

3. we check $A_{\nsubseteq}$ for emptiness.

To construct $A_{M_{L}}$, we observe that a word $w$ is in $q_{s}\left[M_{L}\right]$ if there is a word $a_{1} \cdots a_{n} \in q_{s}$, and for $i \in\{1, \ldots, n\}$, words $w_{i} \in \mathcal{L}\left(G_{a_{i}}\right)$ such that $w=w_{1} \cdots w_{n}$. Hence, $A_{M_{L}}$ simulates $A_{s}$ while accepting words in $\Sigma_{t}^{\prime}$ that are concatenations of words in the various languages $\mathcal{L}\left(G_{a_{i}}\right)$. Specifically, $A_{M_{L}}=\left(\Sigma_{t}^{\prime}, S_{M_{L}}, s_{M_{L}}^{0}, \delta_{M_{L}}, F_{M_{L}}\right)$, where

- $S_{M_{L}}=\Sigma_{s}^{\prime} \times S_{s} \times \mathcal{G}$;

- $s_{M_{L}}^{0}=\Sigma_{s}^{\prime} \times\left\{s_{s}^{0}\right\} \times G_{t}^{\varepsilon}$;

- $F_{M_{L}}=\left\{\left(a, s, G_{a}\right) \mid s \in F_{s}, a \in \Sigma_{s}\right\} \cup$ $\left\{\left(b, s, G_{t}^{b}\right) \mid s \in F_{s}, b \in \Sigma_{n}\right\}$;

- and for each $a \in \Sigma_{s}^{\prime}, s \in S_{s}, R \in \mathcal{G}$, and $b \in \Sigma_{t}^{\prime}$,

$$
\begin{aligned}
& \delta_{M_{L}}((a, s, R), b)= \\
& \begin{cases}\left\{\left(a, s, R \circ G_{t}^{b}\right)\right\}, & \text { if } a \in \Sigma_{s}, R \neq G_{a} ; \\
\left\{\left(a, s, R \circ G_{t}^{b}\right)\right\} \cup & \\
\bigcup_{a^{\prime} \in \Sigma_{s}^{\prime}, s^{\prime} \in \delta_{s}(s, a)}\left\{\left(a^{\prime}, s^{\prime}, G_{t}^{b}\right)\right\}, & \text { if } a \in \Sigma_{s}, R=G_{a} ; \\
\bigcup_{a^{\prime} \in \Sigma_{s}^{\prime}, s^{\prime} \in \delta_{s}(s, a)}\left\{\left(a^{\prime}, s^{\prime}, G_{t}^{\varepsilon}\right)\right\}, & \text { if } a \in \Sigma_{n}, a=b ; \\
\emptyset, & \text { otherwise. }\end{cases}
\end{aligned}
$$

Theorem 21. MSimp[Sound,LAV,RPQ,RPQ] is in PSPaCe. Proof. By Lemma 20, to check whether MSimP[SOUND,LAV,RPQ,RPQ] admits a solution, it suffices guess for each symbol $a \in \Sigma_{s}$ a binary relation $G_{a}$ over the state set $S_{t}$ of $A_{t}$, and check whether for the resulting RPQ-based LAV mapping $M_{L}$ conditions (1) and (2) hold. When checking condition (1), the emptiness test in item (1) can be done for each $a \in \Sigma_{s}$ in NLOGSpACE in $\left|A_{G_{a}}\right|$, and since the number of states of $A_{G_{a}}$ is exponential in $\left|A_{t}\right|$, in PSPACE in $\left|A_{t}\right|$. The non-emptiness test in item (3) can be done in NLOGSPACE in $\left|A_{s}\right|$. When checking condition (2), we do not need to construct $A_{M_{L}}, \bar{A}_{t}$, and $A_{\nsubseteq}$ explicitly, but can check the nonemptiness of $A_{\not \nsubseteq}$ on the fly while constructing $A_{M_{L}}$ and complementing $A_{t}$. Hence, since the number of states of $A_{M_{L}}$ is linear in $\left|A_{s}\right|$ and exponential in $\left|A_{t}\right|$, we get that condition (2) can be checked in PSPACE in $\left|A_{t}\right|$ and in NLogSpace in $\left|A_{s}\right|$.

\subsection{Upper bounds for exact mappings}

The method based on congruence classes can be adapted to address also LAV simplification for exact mappings. The difference wrt sound mappings is that in this case we need to consider also LAV mappings in which the queries are unions of congruence classes. Indeed, congruence classes (and hence solutions to the LAV mapping synthesis problem) are not closed under union, as shown by the following example.

Let $q_{s}=a_{1} \cdot a_{2}$ and $q_{t}=00+01+10$. Then the following two incomparable mappings are solutions to MAXMSYNT[SOUND,LAV, RPQ,RPQ] when the input mapping is $\left\{q_{s} \leadsto q_{t}\right\}$ :

$$
\begin{aligned}
& M_{L}^{1}\left(a_{1}\right)=0, \\
& M_{L}^{1}\left(a_{2}\right)=0+1 . \\
& M_{L}^{2}\left(a_{1}\right)=0+1, \\
& M_{L}^{2}\left(a_{2}\right)=0 .
\end{aligned}
$$

Notice that the mapping $M_{L}$, where $M_{L}\left(a_{i}\right)=M_{L}^{1}\left(a_{i}\right)+$ $M_{L}^{2}\left(a_{i}\right)$, for $i \in\{1,2\}$, is not a solution, since $q_{s}\left[M_{L}\right]$ includes 11 .

On the other hand, we can show that considering mapping in which the queries are unions of congruence classes is 
sufficient to obtain maximal unfoldings. We first generalize Lemma 19 to non-singleton queries.

LEMMA 22. Let $q_{s}$ be an $R P Q$ over $\Sigma_{s}^{\prime}, q_{t}$ an $R P Q$ over $\Sigma_{t}^{\prime}$ expressed through an $1 N F A A_{t}$, and $M_{L}$ a LAV mapping such that $q_{s}\left[M_{L}\right] \subseteq q_{t}$. Then for $M_{L}^{\prime}$ with

$$
M_{L}^{\prime}(a)= \begin{cases}\left.\bigcup_{w \in M_{L}(a)}[w]\right]_{t}, & \text { if } M_{L}(a) \neq \emptyset \\ \emptyset, & \text { if } M_{L}(a)=\emptyset .\end{cases}
$$

we have that $q_{s}\left[M_{L}^{\prime}\right] \subseteq q_{t}$.

Proof. Consider a word $a_{1} \cdots a_{h} \in q_{s}$. If there is one of the $a_{i}$ such that $M_{L}\left(a_{i}\right)=\emptyset$, then $M_{L}\left(a_{1}\right) \cdots M_{L}\left(a_{h}\right)=$ $\emptyset \subseteq q_{t}$. Otherwise, we have that, for $i \in\{1, \ldots, h\}$, for some $w^{a_{i}} \in M_{L}\left(a_{i}\right)$, the word $w^{a_{1}} \cdots w^{a_{h}} \in q_{s}\left[M_{L}\right] \subseteq$ $\mathcal{L}\left(A_{t}\right)$. We show that, for each $i \in\{1, \ldots, h\}$, we also have that $w^{a_{1}} \cdots w^{a_{i-1}} \cdot w^{\prime} \cdot w^{a_{i+1}} \cdots w^{a_{h}} \in \mathcal{L}\left(A_{t}\right)$, for each $w^{\prime} \in \bigcup_{w \in M_{L}\left(a_{i}\right)}[w]_{A_{t}}$. First, since $q_{s}\left[M_{L}\right] \subseteq q_{t}$, if $w^{a_{1}} \cdots w^{a_{h}} \in q_{s}\left[M_{L}\right] \subseteq \mathcal{L}\left(A_{t}\right)$, then, for each $w \in M_{L}\left(a_{i}\right)$, we also have that $w^{a_{1}} \cdots w^{a_{i-1}} \cdot w \cdot w^{a_{i+1}} \cdots w^{a_{h}} \in q_{s}\left[M_{L}\right] \subseteq$ $\mathcal{L}\left(A_{t}\right)$. Then there is a sequence $s_{0}, s_{1}, \ldots, s_{h}$ of states of $A_{t}$ such that $s_{0}=s_{t}^{0}, s_{h} \in F_{t}, s_{j} \in \delta_{t}\left(s_{j-1}, w^{a_{j}}\right)$, for $j \in\{1, \ldots, i-1, i+1, \ldots, h\}$, and $s_{i} \in \delta_{t}\left(s_{i-1}, w\right)$. Then, by the definition of congruence classes, for each word $w^{\prime} \in[w]_{A_{t}}$, we have that $s_{i} \in \delta_{t}\left(s_{i-1}, w_{i}^{\prime}\right)$, and hence $w^{a_{1}} \cdots w^{a_{i-1}} \cdot w^{\prime} \cdot w^{a_{i+1}} \cdots w^{a_{h}} \in \mathcal{L}\left(A_{t}\right)$.

The above lemma implies that, when searching for maximal LAV mappings that imply a given mapping, we can restrict the attention to queries that are unions of congruence classes.

LEMMA 23. Given a mapping $M=\left\{q_{s} \leadsto q_{t}\right\}$, where $q_{t}$ is defined by a 1NFA $A_{t}$, every solution $M_{L}$ to MAXMSYNT[SOUND,LAV,RPQ,RPQ] with input $M$ is such that each query in $M_{L}$ is a union of congruence classes for $A_{t}$.

Proof. Consider a solution $M_{L}$ to MAXMSYNT[SOUND,LAV,RPQ,RPQ] with input $M$, and assume that for some $a \in \Sigma_{s}, M_{L}(a)$ is not a union of congruence classes for $A_{t}$. Then there is some word $w \in M_{L}(a)$ and some word $w^{\prime} \in[w]_{A_{t}}$ such that $w^{\prime} \notin M_{L}(a)$. By Lemma 22, the mapping $M_{L}^{\prime}$ with $M_{L}^{\prime}(a)=M_{L}(a) \cup\left\{w^{\prime}\right\}$ is also a solution to MAXMSYNT[SOUND,LAV,RPQ,RPQ] with input $M$, thus contradicting the maximality of $M_{L}$.

We get the following upper bound for the LAV simplification in the exact case.

Theorem 24. MSimp[EXACT,LAV,RPQ,RPQ] is in EXPSPACE.

Proof. By Lemma 23, we can nondeterministically choose mappings $M_{L}$ in which the queries are unions of congruence classes and then test whether $q_{t}=q_{s}\left[M_{L}\right]$. To do so, we build a 1NFA $A_{s, M_{L}}$ accepting $q_{s}\left[M_{L}\right]$ as follows. We start by observing that for each union $U$ of congruence classes, we can build the automaton $A_{U}=\left(\mathcal{G}, s_{t}, R_{\epsilon}, \delta_{A_{t}}, U\right)$ accepting the words in $U$, which incidentally, is deterministic. Hence, by substituting each $a$-transition in the 1NFA $A_{s}$ for $q_{s}$ with the 1NFA $A_{U_{a}}$, where $M_{L}(a)=U_{a}$, we obtain a 1 NFA $A_{s, M_{L}}$. Note that, even when $A_{s}$ is deterministic, $A_{s, M_{L}}$ may be nondeterministic.
To test $q_{s}\left[M_{L}\right] \subseteq q_{t}$, we complement $A_{t}$, obtaining the $1 \mathrm{NFA} \overline{A_{t}}$, and check the 1NFA $A_{s, M_{L}} \times \overline{A_{t}}$ for emptiness. The size of $A_{s, M_{L}} \times \overline{A_{t}}$ is polynomial in the size of $A_{s}$ and exponential in the size of $A_{t}$. Checking for emptiness can be done in exponential time, and considering the initial nondeterministic guess, we get a NExPTIME upper bound.

To test $q_{t} \subseteq q_{s}\left[M_{L}\right]$, we complement $A_{s, M_{L}}$, obtaining the 1NFA $\overline{A_{s, M_{L}}}$, and check $A_{t} \times \overline{A_{s, M_{L}}}$ for emptiness. Since $A_{s, M_{L}}$ is nondeterministic, complementation is exponential. However, we observe again that such a complementation can be done on the fly in ExpSPACE, while checking for emptiness and intersecting with $A_{t}$. As a consequence, considering the initial nondeteministic guess, MSimP[EXACT,LAV, RPQ,RPQ] can be decided in NEXPSPACE, which is equivalent to ExPSPACE.

Note that the proofs of Theorems 21 and 24 imply that, wrt LAV mapping simplification, considering queries that are RPQs (as opposed to general, possibly non-regular, path languages) is not a restriction, since the existence of general LAV mappings implies the existence of regular ones. This is also in line with a similar observation holding for the existence of rewritings of RPQs wrt RPQ views [21]

Finally, we observe that using the machinery based on unions of congruence classes, we can also solve the maximal mapping synthesis problem. We guess a mapping and check that it is a solution to mapping synthesis. To check that it is a maximal solution, we generate all other mappings and check that they are contained in our candidate solution.

ThEOREM 25. A solution to MAXMSynt[LAV,SOUND, $\mathrm{RPQ}, \mathrm{RPQ}$ and to MAXMSYNT[LAV, EXACT, RPQ,RPQ] can be computed in EXPSPACE.

\subsection{Lower bounds}

It turns out that the upper bound established for the sound case is tight:

Theorem 26. MSimp[SOUnd,LAV,RPQ,RPQ] is PSPACEhard.

Proof. The proof is by a reduction from the universality problem for REs. Given an RE $e$ over the alphabet $\Sigma_{t}=\left\{b_{1}, \ldots, b_{n}\right\}$, let $\Sigma_{s}=\left\{a_{1}, \ldots, a_{n}\right\}$, and let $M_{e}$ be the mapping constituted by the following assertions:

$$
\begin{gathered}
\Sigma_{s}^{*} \leadsto e \\
a_{1} \leadsto b_{1} \quad \cdots \quad a_{n} \leadsto b_{n}
\end{gathered}
$$

We show that $e$ is universal iff MSImP[SOUND,LAV,RPQ,RPQ] with input $M_{e}$ admits a solution. For the "only-if" part, assume that $e$ is universal and consider the LAV mapping $M_{L}$ consisting of the mapping assertions (6). We have that $\Sigma_{s}^{*}\left[M_{L}\right]=\Sigma_{t}^{*}$, and since $e$ is universal, also $\Sigma_{s}^{*}\left[M_{L}\right] \subseteq e$. For the "if-part", consider a LAV mapping $M_{L}$ such that $q_{s}\left[M_{L}\right] \subseteq q_{t}$ and $q_{s}\left[M_{L}\right] \neq \emptyset$, for each mapping assertion $q_{s} \leadsto q_{t}$ in $M_{e}$. By the mapping assertions (6), we have that $M_{L}\left(a_{i}\right) \neq \emptyset$ (since $a_{i}$ is the left-hand side of a mapping assertion) and that $M_{L}\left(a_{i}\right)$ must include $b_{i}$, for $i \in\{1, \ldots, n\}$, and hence $\Sigma_{s}^{*}\left[M_{L}\right]=\Sigma_{t}^{*}$. Since $\Sigma_{s}^{*}\left[M_{L}\right] \subseteq e$, we have that $e$ is universal.

It is easy to see that the above proof shows also PSPACEhardness of simplification for exact mappings. 

PSPACE-hard.

However, we can get a tight lower bound for a generalization of MSIMP [EXACT,LAV,RPQ,RPQ] in which we allow the input mapping to contain both sound and exact mapping assertions. We do so by showing an EXPSPACE lower bound for a problem that is closely related to the mapping simplication problem.

Consider a finite alphabet $\Sigma$ and a finite set $\mathcal{V}$ of variables. A language constraint is a statement of the form $e_{1} \sqsubseteq e_{2}$, where $e_{1}$ and $e_{2}$ are regular expressions over $\Sigma \cup \mathcal{V}$. A language-constraint problem $P$ is a finite set of language constraints. A solution to $P$ is an assignment $\sigma: \mathcal{V} \rightarrow 2^{\Sigma^{*}}$, assigning a language over $\Sigma$ to each variable in $\mathcal{V}$ such that $\mathcal{L}\left(e_{1}[\sigma]\right)=\mathcal{L}\left(e_{2}[\sigma]\right)$. It is easy to express the universality problem for context-free grammars as a language-constraint problem, which implies that the latter is undecidable. Here we consider left-handed language constraint problems, where we allow constraints of the form $e_{1} \sqsubseteq e_{2}$ and $e_{1}=e_{2}$, but require that variables appear only in the left-hand side of the constraint. The technique for the exact version of the LAV mapping simplification problem can be used to show an EXPSPACE upper bound for solving left-handed language constraint problems. We now show a matching lower bound.

To prove the result we exploit a reduction from tiling problems $[47,16]$. A tile is a unit square of one of several types and the tiling problem we consider is specified by means of a finite set $\Delta$ of tile types, two binary relations $H$ and $P$ over $\Delta$, representing horizontal and vertical adjacency relations, respectively, and two distinguished tile types $t_{S}, t_{F} \in \Delta$. The tiling problem consists in determining whether, for a given number $n$ in unary, a region of the integer plane of size $2^{n} \times k$, for some $k>0$, can be tiled consistently with the adjacency relations $H$ and $P$, and with the left bottom tile of the region of type $t_{S}$ and the right upper tile of type $t_{F}$. We also require that the last tile of a row and the first tile of the next row are consistent with $H$. Using a reduction from acceptance of EXPSPACE Turing machines analogous to the one in [47], it can be shown that this tiling problem is EXPSPACE-complete.

THEOREM 28. Solving left-handed language constraints is EXPSPACE-complete.

Proof SKeTCh. We sketch the lower-bound argument.

Let $T=\left(\Delta, H, P, t_{S}, t_{F}\right)$ be an instance of the ExPSPACEcomplete tiling problem above and $n$ a number in unary. The alphabet is $\Sigma=\Delta \cup\{0,1\}^{3} \cup\{\#\}$. Intuitively, the letters in $\Delta$ denote tiles, symbols in $\{0,1\}^{3}$ denote address bits, and \# denotes a separation marker. The idea is to encode each tiled cell by a word of length $n+2$ of the form $\# \cdot\left(\{0,1\}^{3}\right)^{n} \cdot \Delta$, consisting of a marker, an $n$-bit address, and a tile symbol. We use an element in $\{0,1\}^{3}$ for each address bit to make it easy to check that two $n$-bit addresses are consecutive; we use $n$-bits for the current address, $n$ bits for the carry, and $n$ bits for the next address. Thus, each tiling can be described by a word in $\left(\# \cdot\left(\{0,1\}^{3}\right)^{n} \cdot \Delta\right)^{*}$, obtained by encoding each cell as described above, and then concatenating the symbols, first column by column and then row by row.

Consider a word $w \in \Sigma^{*}$. Such a word does not describe a proper tiling if one of the following errors can be found in the word:

1. The symbol \# does not occur precisely in positions $(n+2) i$, for $i=0,1, \ldots$
2. The symbols in $\Delta$ do not occur precisely in positions $n+1+(n+2) i$, for $i=0,1, \ldots$.

3. The first address is not $0^{n}$.

4. The last address is not $1^{n}$.

5. There is a pair of adjacent but not successive addresses.

6. The first tile is not $t_{S}$.

7. The last tile is not $t_{F}$.

8. There is a pair of adjacent blocks with tiles that violate the relation $H$.

9. There is a pair of vertically adjacent blocks with tiles that violate the relation $P$.

We do need to define the notion of vertical adjacency. Two blocks are vertically adjacent if their addresses agree and either both addresses are $0^{n}$ and there is no occurrence of $0^{n}$ between them, or both addresses are not $0^{n}$ and there is precisely one ocurrence of $0^{n}$ between them.

If the tiling problem has no solution, then every word in $\Sigma^{*}$ must contain an error. We now define a constraint of the form $e_{\text {error }}=\Sigma^{*}$, where the "task" of $e_{\text {error }}$ is to discover errors in candidate words. The expression $e_{\text {error }}$ is the sum of several terms corresponding to the various errors. We now sketch how to "discover" these possible errors. In order to have the left-hand sides use only variables, we introduce a variable $v_{a}$ for each letter $a \in \Sigma$, accompanied by the constraint $v_{a}=a$. We use $\mathcal{V}_{\Sigma}$ to abbreviate $\sum_{a \in \Sigma} v_{a}$.

Most of the errors can be discovered with a single regular expression. For example, the error where the symbol \# does not occur precisely in positions $(n+2) i$, for $i=0,1, \ldots$, is described using the expression

$$
\left(\mathcal{V}_{\Sigma}^{n+2}\right)^{*} \cdot\left(\sum_{1 \leq i \leq n+1} \mathcal{V}_{\Sigma}^{i}\right) \cdot \# \cdot \mathcal{V}_{\Sigma}^{*}
$$

The one error that is challenging is where there is a pair of vertically adjacent blocks with tiles that violate the relation $P$. Discovering this error is more difficult and cannot be done by one regular expression; rather, several additional constraints are needed. For simplicity we ignore the fact that each address bit is encoded by three bits rather than one.

Let $e_{n z a}$ be a regular expression that describes nonzero addresses: $\sum_{0 \leq i \leq n-1}\{0,1\}^{i} \cdot 1 \cdot\{0,1\}^{n-i-1}$.

We add to $e_{\text {error }}$ the following term, which discovers nonmatching tiles at zero-addressed vertically adjacent tiles.

$$
\sum_{\left(t, t^{\prime}\right) \notin P}\left(\mathcal{V}_{\Sigma}^{*} \cdot \# \cdot 0^{n} \cdot t \cdot\left(\# \cdot e_{n z a} \cdot \Delta\right)^{*} \cdot \# \cdot 0^{n} \cdot t^{\prime} \cdot \mathcal{V}_{\Sigma}^{*}\right)
$$

We need to deal with non-zero-addessed vertically adjacent blocks. For this we use several constraints. First:

$$
\begin{gathered}
v_{n z a v a} \sqsubseteq \sum_{\left(t, t^{\prime}\right) \notin P}\left(\# \cdot e_{n z a} \cdot t \cdot\left(\# \cdot e_{n z a} \cdot \Delta\right)^{*} \cdot \# \cdot 0^{n} \cdot \Delta \cdot\right. \\
\left.\left(\# \cdot e_{n z a} \cdot \Delta\right)^{*} \cdot \# \cdot e_{n z a} \cdot t^{\prime}\right)
\end{gathered}
$$

This says that $v_{n z a v a}$ describes sequences of blocks that start and end with a pair of non-matching non-zeroaddressed blocks, with a single zero-addressed block in between. We still have to impose the constraint that the first and last block have equal addresses. We do this with $n$ constraints, one for each bit of the address. That is for each $i$, $0 \leq i \leq n-1$, we add the constraint:

$$
\begin{aligned}
v_{\text {nzava }} \sqsubseteq\left(\# \cdot\{0,1\}^{i} \cdot 0 \cdot\{0,1\}^{n-i-1} \cdot \Delta \cdot\left(\# \cdot\{0,1\}^{n} \cdot \Delta\right)^{*} .\right. \\
\left.\# \cdot\{0,1\}^{i} \cdot 0 \cdot\{0,1\}^{n-i-1} \cdot \Delta\right)+ \\
\left(\# \cdot\{0,1\}^{i} \cdot 1 \cdot\{0,1\}^{n-i-1} \cdot \Delta \cdot\left(\# \cdot\{0,1\}^{n} \cdot \Delta\right)^{*} .\right. \\
\left.\quad \# \cdot\{0,1\}^{i} \cdot 1 \cdot\{0,1\}^{n-i-1} \cdot \Delta\right)
\end{aligned}
$$

This constraint says that the $i$-th bits of the first and last addresses are either both 0 or both 1 . 
Now we can add to $e_{\text {error }}$ the term $\mathcal{V}_{\Sigma}^{*} \cdot v_{\text {nzava }} \cdot \mathcal{V}_{\Sigma}^{*}$, which discovers all errors due to not-matching, non-zero-addressed vertically adjacent blocks.

Note that the constraint system constructed is of size quadratic in the size of the tiling system. If the tiling problem has no solution, then every word in $\Sigma^{*}$ contains an error and the contraint problem constructed is satisfiable. If the tiling problem has a solution, then a word describing a proper tiling has no error, and for no assignment $\sigma: \mathcal{V} \rightarrow 2^{\Sigma^{*}}$ we have $\mathcal{L}\left(e_{\text {error }}[\sigma]\right)=\Sigma^{*}$, since $e_{\text {error }}$ captures only errors.

Let MSimp[MIXED,LAV,RPQ,RPQ], be the following decision problem: given an RPQ-based schema mapping $M$ consisting both of SOUND and of EXACT mapping assertions, check whether there exists an RPQ-based LAV schema mapping $M^{\prime}$ of type EXACT such that $M^{\prime} \mid=M$, and $M^{\prime} \not \neq_{\text {triv }} M$.

As a corollary of Theorem 28, we get the following result.

Corollary 29. MSimp[MIXED,LAV,RPQ,RPQ]

EXPSPACE-complete.

\section{EXTENSIONS}

In this section we sketch the extension of the results of the previous section on simplification in terms of LAV mappings to more expressive classes of queries: 2RPQs, CRPQs, UCRPQs, and UC2RPQs.

\subsection{RPQs}

Consider now simplification for mappings based on 2RPQs, expressed by means of 1NFAs over the alphabets $\Sigma_{s}^{ \pm}$and $\Sigma_{t}^{ \pm}$.

A key concept for 2RPQs is that of folding, of a language [22], which intuitively denotes the set of words that are the result of repeatedly cancelling out adjacent occurrences of a symbol and its inverse. Let $u, v \in \Sigma^{ \pm}$. We say that $v$ folds onto $u$, denoted $v \rightsquigarrow u$, if $v$ can be "folded" on $u$, e.g., $a b b^{-} b c \rightsquigarrow a b c$. Formally, we say that $v=v_{1} \cdots v_{m}$ folds onto $u=u_{1} \cdots u_{n}$ if there is a sequence $i_{0}, \ldots, i_{m}$ of positive integers between 0 and $|u|$ such that

- $i_{0}=0$ and $i_{m}=n$, and

- for $j \in\{0, \ldots, m\}$, either $i_{j+1}=i_{j}+1$ and $v_{j+1}=$ $u_{i_{j}+1}$, or $i_{j+1}=i_{j}-1$ and $v_{j+1}=u_{i_{j+1}}^{-}$.

Let $L$ be a language over $\Sigma^{ \pm}$. We define fold $(L)=\{u \mid v \rightsquigarrow$ $u, v \in L\}$.

A language-theoretic characterization for containment of 2RPQs was provided in [22]:

LEMMA 30. Let $q_{1}$ and $q_{2}$ be 2RPQs. Then $q_{1} \sqsubseteq q_{2}$ iff $\mathcal{L}\left(q_{1}\right) \subseteq \operatorname{fold}\left(\mathcal{L}\left(q_{2}\right)\right)$.

Furthermore, it is shown in [22] that if $A$ is an $n$-state 1NFA over $\Sigma^{ \pm}$, then there is a $2 \mathrm{NFA}$ for $\operatorname{fold}(\mathcal{L}(A))$ with $n \cdot\left(\left|\Sigma^{ \pm}\right|+\right.$ 1 ) states. (We use $2 \mathrm{NFA}$ to refer to two-way automata.)

In the mapping simplification problem, we are given queries $q_{s}$ and $q_{t}$, expressed as 1NFAs $A_{s}$ and $A_{t}$, respectively, and we are asked whether there exist a 2RPQ-based LAV mapping $M_{L}$ such that $q_{s}\left[M_{L}\right] \sqsubseteq q_{t}$ or $q_{s}\left[M_{L}\right]=q_{t}$, and also $q_{s}[V] \not \equiv \emptyset$.

Here we can still use Lemma 23 for the congruence-class based solution. A simplistic approach would be to convert the 2NFA for fold $\left(\mathcal{L}\left(A_{t}\right)\right)$ into a 1NFA, with an exponential blow-up, and proceed as in Section 5. To avoid this exponential blowup, we need an exponential bound on the number of congruence classes. For a 1NFA, we saw that each congruence class can be defined in terms of a binary relation over its set of states. It turns out that for a $2 \mathrm{NFA}$ $A$, a congruence class can be defined in terms of four binary relations over the set $S_{t}$ of states of $A$ :

1. $R_{l r}$ : a pair $\left(s_{1}, s_{2}\right) \in R_{l r}$ means that there is a word $w$ that leads $A$ from $s_{1}$ to $s_{2}$, where $w$ is entered on the left and exited on the right.

2. $R_{r l}$ : a pair $\left(s_{1}, s_{2}\right) \in R_{r l}$ means that there is a word $w$ that leads $A$ from $s_{1}$ to $s_{2}$, where $w$ is entered on the right and exited on the left.

3. $R_{l l}$ : a pair $\left(s_{1}, s_{2}\right) \in R_{l l}$ means that there is a word $w$ that leads $A$ from $s_{1}$ to $s_{2}$, where $w$ is entered on the left and exited on the left.

4. $R_{r r}$ : a pair $\left(s_{1}, s_{2}\right) \in R_{r r}$ means that there is a word $w$ that leads $A$ from $s_{1}$ to $s_{2}$, where $w$ is entered on the right and exited on the right.

Thus, the number of congruence classes when $A$ has $m$ states is $2^{4 m^{2}}$ rather than $2^{m^{2}}$, which is still an exponential. This enables us to adapt the technique of Section 5 with essentially the same complexity bounds.

THEOREM 31. MSimp[SOUND,LAV,2RPQ,2RPQ] is PSPACE-complete. MSIMP[EXACT, LAV,2RPQ,2RPQ] is in EXPSPACE.

\subsection{CRPQs and UC2RPQs}

Consider now the mapping simplification problem for the case where the input mapping is expressed in terms of CRPQs, where the constituent RPQs are expressed by means of 1NFAs. Here the LAV mappings have to be in terms of RPQs, rather than CRPQs, since CRPQs are not closed under substitutions. The crux of our approach is to reduce containment of two CRPQs, $q_{1}$ and $q_{2}$ to containment of standard languages. This was done in [19]. Let $q_{h}$, for $h=\{1,2\}$, be in the form

$$
q_{h}=\left\{\left(x_{1}, \ldots, x_{n}\right) \mid \begin{array}{l}
q_{h, 1}\left(y_{h, 1}, y_{h, 2}\right) \wedge \cdots \wedge \\
\\
\left.q_{h, m_{h}}\left(y_{h, 2 m_{h}-1}, y_{h, 2 m_{h}}\right)\right\}
\end{array}\right.
$$

and let $\mathcal{V}_{1}, \mathcal{V}_{2}$ be the sets of variables of $q_{1}$ and $q_{2}$ respectively. It is shown in [19] that the containment $q_{1} \sqsubseteq q_{2}$ can be reduced to the containment $\mathcal{L}\left(A_{1}\right) \subseteq \mathcal{L}\left(A_{2}\right)$ of two word automata $A_{1}$ and $A_{2} . A_{1}$ is a $1 \mathrm{NFA}$, whose size is exponential in $q_{1}$ and it accepts certain words of the form

$$
\$ d_{1} w_{1} d_{2} \$ d_{3} w_{2} d_{4} \$ \cdots \$ d_{2 m_{1}-1} w_{m_{1}} d_{2 m_{1}} \$
$$

where each $d_{i}$ is a subset of $\mathcal{V}_{1}$ and the words $w_{i}$ are over the alphabet of $A_{1}$. Such words constitute a linear representation of certain graph databases that are canonical for $q_{1}$ in some sense. $A_{2}$ is a $2 \mathrm{NFA}$, whose size is exponential in the size of $q_{2}$, and it accepts words of the above form if the there is an appropriate mapping from $q_{2}$ to the database represented by these words. The reduction of the containment $q_{1} \sqsubseteq q_{2}$ to $\mathcal{L}\left(A_{1}\right) \subseteq \mathcal{L}\left(A_{2}\right)$ is shown in [19].

The ability to reduce containment of CRPQs to containment of word automata means that we can also apply the congruence-class technique of Section 5. Suppose that we have an RPQ-based LAV mapping $M_{L}$ such that $\mathcal{L}\left(A_{s}\left[M_{L}\right]\right) \subseteq \mathcal{L}\left(A_{t}\right)$. Then we can again assume that the 
queries in the LAV mapping are closed with respect to the congruence classes of $A_{t}$. Thus, the techniques of Section 5 can be applied.

THEOREM 32. MSimP[SOUND,LAV,CRPQ,RPQ] is in EXPSPACE. MSimp[EXACT, LAV, CRPQ,RPQ] is in 2EXPSPACE.

Finally, we consider UC2RPQ queries, which combine UCQs and 2RPQs. This requires combining the techniques developed for RPQs, 2RPQs, and CRPQs. The key idea is the reduction of query containment to containment of word automata. The resulting upper bounds are identical to those we obtained for CRPQs.

\section{GAV SIMPLIFICATION}

In this section, we consider the case of simplifying mappings in terms of GAV mappings. Our results cover only a subset of the possible combination of the problem space, specifically GAV simplification is wide open for RPQ-based mappings. It should be noted that for exact mappings, the LAV case and GAV case coincide, so the results from Section 4 apply; we focus therefore on sound mappings.

We start by considering sound CQ-based mappings.

\section{Theorem 33. MSimp[SOUnd, GAV, $\mathrm{CQ}, \mathrm{CQ}]$ is in $N P$.}

Proof. Consider a sound CQ-based mapping $M$ consisting of a single assertion $q_{s} \leadsto q_{t}$ and assume there exists some sound CQ-based GAV mapping $M_{G}$ such that $q_{s} \sqsubseteq q_{t}\left[M_{G}\right]$, witnessed by a homomorphism $h$, and $q_{t}\left[M_{G}\right] \neq$ true $^{\mathcal{D}_{t}}$. We show that there is a sound CQ-based GAV mapping $M_{G}^{\prime}$ of bounded size such that $q_{s} \sqsubseteq q_{t}\left[M_{G}^{\prime}\right]$. Indeed, since $q_{t}\left[M_{G}\right] \neq$ true $^{\mathcal{D}_{t}}$, there must be one distinguished variable $x$ and one symbol $b \in \Sigma_{t}$, such that $x$ occur in an atomic formula with the symbol $b$ in $M_{G}[b]$. We now obtain $M_{G}^{\prime}(b)$ from $M_{G}(b)$ by selecting in $M_{G}(b)$ such an atom. For all other symbols $b^{\prime} \in \Sigma_{t}$, we take $M_{G}^{\prime}\left(b^{\prime}\right)$ to be true. By constructing $M_{G}^{\prime}$ in this way, we have that the atoms in $q_{t}\left[M_{G}^{\prime}\right]$ are a subset of the atoms in $q_{t}\left[M_{G}\right]$, and hence the projection of $h$ on such atoms is still a homomorphism to $q_{s}$.

In the general case where the mapping $M$ consists of $k$ assertions, we can apply the above argument for each of the assertions in $M$. This shows that, if there exists some sound GAV mapping $M_{G}$ such that $M_{G} \models M$, then there is also a GAV mapping $M_{G}^{\prime}$ such that $M_{G}^{\prime}(b)$ has at most $k$ atoms and $M_{G}^{\prime} \models M$. Hence, in order to check the existence of an appropriate GAV mapping $M_{G}$, it suffices to guess (avoiding trivial mappings), for each symbol $b \in \Sigma_{t}$ appearing in $M$, a CQ $M_{G}(b)$ over $\Sigma_{s}$ of size at most $k$, and check that $q_{s} \sqsubseteq$ $q_{t}\left[M_{G}\right]$, for each $q_{s} \sim q_{t} \in M$.

This result extends immediately to UCQ-based mappings, by checking containment between UCQs, instead of CQs.

Theorem 34. MSimp[SOUnd, GAV, $\mathrm{UCQ}, \mathrm{CQ}$ ] is in NP.

For UCQ-based mappings, we get the same upper bound, with a somewhat subtler argument.

\section{Theorem 35. MSimp[SOUnd, GAV, $\mathrm{UCQ}, \mathrm{UCQ}]$ is in NP.}

Proof. We consider first the case of a sound UCQ-based mapping $M$ consisting of a single assertion $q_{s} \leadsto q_{t}$. Assume there exists some sound UCQ-based GAV mapping $M_{G}$ such that $q_{s} \sqsubseteq q_{t}\left[M_{G}\right]$ and $q_{t}\left[M_{G}\right] \neq$ true $^{\mathcal{D}_{t}}$. First note that $q_{s} \sqsubseteq$
$q_{t}\left[M_{G}\right]$, if for each CQ $q_{s}^{i}$ of $q_{s}$ we have that $q_{s}^{i} \sqsubseteq q_{t}\left[M_{G}\right]$. This means that there is a CQ $q_{t}^{i}$ of $q_{t}$ and a CQ $q_{b}^{i}$ for each symbol $b \in \Sigma_{t}$ such that there is a homomorphism from $q_{t}^{i}\left[M_{G}^{\prime}\right]$ to $q_{s}^{i}$, where $M_{G}^{\prime}(b)=q_{b}^{i}$. Thus, if $q_{s}$ is a union of $l$ CQs, then we can assume that each $M_{G}[b]$ for $b \in \Sigma_{t}$ has at most $l$ CQs. What is left is to bound the size of these CQs.

Let $m$ be the number of CQs in $q_{t}$. Since $q_{t}\left[M_{G}\right] \neq$ true $^{\mathcal{D}_{t}}$, for each CQ $q_{t}^{\prime}$ of $q_{t}$, there must be one distinguished variable $x$ and one symbol $b \in \Sigma_{t}$ such that $x$ occurs in some atomic formula with the symbol $b$ in each CQ $q_{b}^{\prime}$ of $M_{G}[b]$. Define $M_{G}^{\prime}$ to keep all such atomic formulas, and only such atomic formulas. $M_{G}^{\prime}[b]$ contains at most $l m$ atomic formulas. If we have $k$ mapping assertions, then $M_{G}^{\prime}[b]$ needs to contain only $\mathrm{klm}$ atomic formulas. To check the existence of a simplifying GAV mapping it suffices to guess a mapping $M_{G}^{\prime}$ under such a size bound and check that $q_{s} \sqsubseteq q_{t}\left[M_{G}^{\prime}\right]$.

We conjecture that the above upper bounds are tight.

\section{CONCLUSIONS}

We have introduced the problem of simplifying schema mappings based on logical implication. The problem comes in different forms, depending on the type of simplification to achieve, on whether the mappings are sound or exact, and on the types of queries used in the mappings. We have provided a formalization of the problem, and we have presented techniques and complexity bounds for both relational and graph databases. As we said in the introduction, this is the first investigation on comparing schema mappings for graph databases.

In this paper we have concentrated on LAV simplification, and we have discussed the GAV case only for relational schema mappings. In the future, we plan to continue investigating schema mapping simplification along different directions. In particular, we aim at addressing GAV simplification for graph databases, and we plan to study schema mapping simplification for tree-based (e.g., XML) semistructured data.

\section{Acknowledgements}

Work partially supported by the EU under the project ACSI (Artifact-Centric Service Interoperation), grant n. FP7257593 , by Regione Lazio under the project "Integrazione semantica di dati e servizi per le aziende in rete", and by NSF grants CCF-0613889, CCF-0728882, and CNS 1049862.

\section{REFERENCES}

[1] S. Abiteboul. Querying semi-structured data. In Proc. of ICDT'97, pages 1-18.

[2] S. Abiteboul, P. Buneman, and D. Suciu. Data on the Web: from Relations to Semistructured Data and $X M L$. Morgan Kaufmann, 2000.

[3] S. Abiteboul and O. Duschka. Complexity of answering queries using materialized views. In Proc. of PODS'98, pages 254-265.

[4] S. Abiteboul, G. Gottlob, and M. Manna. Distributed XML design. In Proc. of PODS 2009, pages 247-258.

[5] S. Abiteboul, R. Hull, and V. Vianu. Foundations of Databases. Addison Wesley Publ. Co., 1995.

[6] B. Alexe, P. Kolaitis, and W.-C. Tan. Characterizing schema mappings via data examples. In Proc. of PODS 2010, pages 261-271. 
[7] M. Arenas, P. Barcelo, R. Fagin, and L. Libkin. Locally consistent transformations and query answering in data exchange. In Proc. of PODS 2004.

[8] M. Arenas, R. Fagin, and A. Nash. Composition with target constraints. In Proc. of ICDT 2010.

[9] M. Arenas and L. Libkin. XML data exchange: Consistency and query answering. In Proc. of PODS 2005, pages 13-24.

[10] M. Arenas, J. Pérez, J. L. Reutte, and C. Riveros. Foundations of schema mapping management. In Proc. of PODS 2010, pages 227-238.

[11] M. Arenas, J. Pérez, and C. Riveros. The recovery of a schema mapping: Bringing exchanged data back. ACM Trans. on Database Systems, 34(4), 2009.

[12] P. C. Arocena, A. Fuxman, and R. J. Miller. Composing local-as-view mappings: Closure and applications. In Proc. of ICDT 2010, pages 209-218.

[13] F. Baader and R. Küsters. Unification in a description logic with transitive closure of roles. In R. Nieuwenhuis and A. Voronkov, editors, Proc. of LPAR 2001, volume 2250 of $L N C S$, pages 217-232. Springer.

[14] B. Barcelò. Logical foundations of relational data exchange. SIGMOD Record, 38(1):49-58, 2009.

[15] B. Barcelò, C. A. Hurtado, L. Libkin, and P. T. Wood. Expressive languages for path queries over graph-structured data. In Proc. of PODS 2009.

[16] R. Berger. The undecidability of the dominoe problem. Mem. Amer. Math. Soc., 66:1-72, 1966.

[17] P. A. Bernstein and H. Ho. Model management and schema mappings: Theory and practices. In Proc. of $V L D B$ 200\%, pages 1439-1440.

[18] P. Buneman. Semistructured data. In Proc. of PODS'97, pages 117-121.

[19] D. Calvanese, G. De Giacomo, M. Lenzerini, and M. Y. Vardi. Containment of conjunctive regular path queries with inverse. In Proc. of KR 2000.

[20] D. Calvanese, G. De Giacomo, M. Lenzerini, and M. Y. Vardi. Query processing using views for regular path queries with inverse. In Proc. of PODS 2000.

[21] D. Calvanese, G. De Giacomo, M. Lenzerini, and M. Y. Vardi. Rewriting of regular expressions and regular path queries. J. of Computer and System Sciences, 64(3):443-465, 2002.

[22] D. Calvanese, G. De Giacomo, M. Lenzerini, and M. Y. Vardi. Reasoning on regular path queries. SIGMOD Record, 32(4):83-92, 2003.

[23] D. Calvanese, G. De Giacomo, M. Lenzerini, and M. Y. Vardi. View synthesis from schema mappings. CoRR Technical Report abs/1003.1179, arXiv.org e-Print archive, Mar. 2010. Available at http://arxiv.org/abs/1003.1179.

[24] A. K. Chandra and P. M. Merlin. Optimal implementation of conjunctive queries in relational data bases. In Proc. of STOC'ryn, pages 77-90.

[25] I. F. Cruz, A. O. Mendelzon, and P. T. Wood. A graphical query language supporting recursion. In Proc. of ACM SIGMOD, pages 323-330, 1987.

[26] R. Fagin, P. G. Kolaitis, R. J. Miller, and L. Popa. Data exchange: Semantics and query answering. Theor. Comp. Sci., 336(1):89-124, 2005.

[27] R. Fagin, P. G. Kolaitis, A. Nash, and L. Popa.
Towards a theory of schema-mapping optimization. In Proc. of PODS 2008, pages 33-42.

[28] R. Fagin, P. G. Kolaitis, and L. Popa. Data exchange: Getting to the core. ACM Trans. on Database Systems, 30(1):174-210, 2005.

[29] R. Fagin, P. G. Kolaitis, L. Popa, and W. C. Tan. Reverse data exchange: Coping with nulls. In Proc. of PODS 2009, pages 23-32.

[30] R. Fagin, P. G. Kolaitis, L. Popa, and W.-C. Tan. Composing schema mappings: Second-order dependencies to the rescue. ACM Trans. on Database Systems, 30(4):994-1055, 2005.

[31] R. Fagin, P. G. Kolaitis, L. Popa, and W.-C. Tan. Quasi-inverses of schema mappings. ACM Trans. on Database Systems, 33(2):1-52, 2008.

[32] E. Franconi and S. Tessaris. The logic of RDF and SPARQL: a tutorial. In Proc. of PODS 2006.

[33] A. Fuxman, P. G. Kolaitis, R. J. Miller, and W. C. Tan. Peer data exchange. ACM Trans. on Database Systems, 31(4):1454-1498, 2005.

[34] G. Gottlob, R. Pichler, and V. Savenkov. Normalization and optimization of schema mappings. PVLDB, 2(1):1102-1113, 2009.

[35] G. Grahne and A. O. Mendelzon. Tableau techniques for querying information sources through global schemas. In Proc. of ICDT'99, volume 1540 of $L N C S$, pages 332-347. Springer.

[36] A. Y. Halevy, A. Rajaraman, and J. Ordille. Data integration: The teenage years. In Proc. of $V L D B$ 2006, pages 9-16.

[37] P. G. Kolaitis. Schema mappings, data exchange, and metadata management. In Proc. of PODS 2005.

[38] M. Lenzerini. Data integration: A theoretical perspective. In Proc. of PODS 2002, pages 233-246.

[39] A. Y. Levy, A. O. Mendelzon, Y. Sagiv, and D. Srivastava. Answering queries using views. In Proc. of PODS'95, pages 95-104.

[40] L. Libkin and C. Sirangelo. Data exchange and schema mappings in open and closed worlds. In Proc. of PODS 2008, pages 139-148.

[41] J. Madhavan and A. Y. Halevy. Composing mappings among data sources. In Proc. of VLDB 2003.

[42] W. Martens, M. Niewerth, and T. Schwentick. Schema design for XML repositories: Complexity and tractability. In Proc. of PODS 2010, pages 239-250.

[43] J.-E. Pin. Syntactic semigroups. In G. Rozenberg and A. Salomaa, editors, Handbook of Formal Language Theory, volume 1, chapter 10, pages 679-746. Springer, 1997.

[44] Y. Sagiv and M. Yannakakis. Equivalences among relational expressions with the union and difference operators. J. of the ACM, 27(4):633-655, 1980.

[45] B. ten Cate and P. G. Kolaitis. Structural characterizations of schema-mapping languages. In Proc. of ICDT 2009, pages 63-72.

[46] J. D. Ullman. Information integration using logical views. In Proc. of ICDT'97, volume 1186 of $L N C S$.

[47] P. van Emde Boas. The convenience of tilings. In Complexity, Logic, and Recursion Theory, volume 187 of Lecture Notes in Pure and Applied Mathematics, pages 331-363. Marcel Dekker Inc., 1997. 


\section{APPENDIX: PROOFS}

\section{Proof of Theorem 9.}

Consider a sound UCQ-based mapping $M$ consisting of a single assertion $q_{s} \leadsto q_{t}$ and a sound CQ-based LAV mapping $M_{L}$ such that $q_{s}\left[M_{L}\right] \sqsubseteq q_{t}$. We remind that $q_{s}\left[M_{L}\right] \sqsubseteq q_{t}$ if for each CQ $q_{1}$ in the UCQ $q_{s}\left[M_{L}\right]$ there is a CQ $q_{2}$ in the UCQ $q_{t}$ such that $q_{1} \sqsubseteq q_{2}$. Then, for each CQ $q_{1}$ in $q_{s}\left[M_{L}\right]$ there exists a homomorphism from some CQ $q_{2}$ in $q_{t}$ to $q_{1}$, and for each $a \in \Sigma_{s}$ at most $\ell_{q_{t}}$ atoms in the query $M_{L}(a)$ are needed for the homomorphism, where $\ell_{q_{t}}$ is the maximum number of atoms among the CQs in $q_{t}$. In the general case where the mapping $M$ consists of several assertions, for each $a \in \Sigma_{s}$ at most $\ell_{M}=\sum_{q_{s} \sim q_{t} \in M} \ell_{q_{t}}$ atoms in the query $M_{L}(a)$ are needed for the homomorphisms for all the assertions in $M$. Thus, in order to check for the existence of an appropriate LAV mapping $M_{L}$, it suffices to guess (again avoiding trivial implication), for each symbol $a \in \Sigma_{s}$ appearing in $M$, a CQ $M_{L}(a)$ over $\Sigma_{t}$ of size at most $\ell_{M}=\sum_{q_{s} \sim q_{t} \in M} \ell_{q_{t}}$, and check for each $q_{s} \leadsto q_{t} \in M$ that $q_{s}\left[M_{L}\right] \sqsubseteq q_{t}$ (and $q_{t} \sqsubseteq q_{s}\left[M_{L}\right]$ for the exact variant).

\section{Proof of Theorem 12.}

Consider an exact UCQ-based mapping $M$ consisting of a single assertion $q_{s} \leadsto q_{t}$ and an exact UCQ-based LAV mapping $M_{L}$ such that $M_{L}=M$. Let $m_{q_{t}}$ be the number of CQs in $q_{t}$, and $\ell_{q_{t}}$ the maximum number of atoms among the CQs in $q_{t}$. Let $q_{s, M_{L}}^{\prime}$ be obtained from $q_{s}\left[M_{L}\right]$ by distributing, for each atom $\alpha$ of $q_{s}$, the unions in the UCQ $\alpha\left[M_{L}\right]$ over the conjunctions of each CQ of $q_{s}$. Note that $q_{s, M_{L}}^{\prime}$ is a UCQ, and is equivalent to $q_{s}\left[M_{L}\right]$. Since $q_{s}\left[M_{L}\right] \sqsubseteq q_{t}$, for each CQ in $q_{s, M_{L}}^{\prime}$, there is a homomorphism from some CQ in $q_{t}$ to it. Hence, for each symbol $a \in \Sigma_{s}$ occurring in $q_{s}$, for each CQ in the UCQ $M_{L}(a)$, we need at most $\ell_{q_{t}}$ atoms for the homomorphism from $q_{t}$. To derive a bound for the number of such CQs, we observe that the inclusion $q_{t} \sqsubseteq q_{s}\left[M_{L}\right]$ must also hold. Therefore, we need at most $m_{q_{t}}$ CQs in $M_{L}(a)$ to satisfy the inclusion of all the CQs in $q_{t}$. It follows that, to check for the existence of the LAV mapping $M_{L}$ and the corresponding homomorphism, it suffices to guess for each $a \in \Sigma_{s}$ a UCQ over $\Sigma_{t}$ consisting of at most $m_{q_{t}}$ CQs, each with at most $\ell_{q_{t}}$ atoms. In the general case where the mapping $M$ consists of several assertions $q_{s} \leadsto q_{t}$, we can proceed analogously to the case above, using instead of $m_{q_{t}}$ and $\ell_{q_{t}}$, the sum of these parameters over all mapping assertions in $M$. To check whether $q_{t} \sqsubseteq q_{s}\left[M_{L}\right]$, it suffices to check for the existence of a homomorphism from $q_{s}\left[M_{L}\right]$ to each of the CQs in $q_{t}$, which can be done in NP in the size of $q_{t}$. To check whether $q_{s}\left[M_{L}\right] \sqsubseteq q_{t}$, we have to check whether for each CQ $q^{\prime}$ obtained by selecting one of the CQs $q^{\prime \prime}$ in $q_{s}$ and then substituting each atom $\alpha$ in $q^{\prime \prime}$ with one of the CQs in $\alpha\left[M_{L}\right]$, there is a homomorphism from some CQ in $q_{t}$ to $q^{\prime}$. We can do so by a coNP computation that makes use of an NP oracle to check for the existence of a homomorphism. This gives us the $\Pi_{2}^{p}$ upper bound.

\section{Proof of Theorem 13}

The proof is by a reduction from 3-colorability. Consider a graph $G=(N, E)$, with $N=\left\{n_{1}, \ldots, n_{k}\right\}$.

$$
\begin{aligned}
& \text { Let } \Sigma_{s}=\left\{t / 2, a_{s} / 2, a_{f} / 2\right\}, \Sigma_{t}=\left\{e / 2, b_{s} / 2, b_{f} / 2\right\} \text {, } \\
& q_{T}=\left\{(s, f) \mid a_{s}(s, r), a_{s}(s, g), a_{s}(s, b)\right. \text {, } \\
& t(r, g), t(g, r), t(r, b), t(b, r), t(g, b), t(b, g) \text {, } \\
& \left.a_{f}(r, f), a_{f}(g, f), a_{f}(b, f)\right\} \\
& q_{G}=\left\{(s, f) \mid b_{s}\left(s, x_{1}\right), \ldots, b_{s}\left(s, x_{k}\right)\right. \text {, } \\
& \bigwedge_{\left(n_{i}, n_{j}\right) \in E}\left\{e\left(x_{i}, x_{j}\right), e\left(x_{j}, x_{i}\right)\right\}, \\
& \left.b_{f}\left(x_{1}, f\right), \ldots, b_{f}\left(x_{k}, f\right)\right\}
\end{aligned}
$$

and define the following mapping $M$ :

$$
\begin{aligned}
q_{T} & \sim q_{G} \\
\{(x, y) \mid t(x, y)\} & \sim\{(x, y) \mid e(x, y)\} \\
\left\{(x, y) \mid a_{s}(x, y)\right\} & \sim\left\{(x, y) \mid b_{s}(x, y)\right\} \\
\left\{(x, y) \mid a_{f}(x, y)\right\} & \sim\left\{(x, y) \mid b_{f}(x, y)\right\}
\end{aligned}
$$

Intuitively, assertion (7) maps a triangle, whose three vertexes are connected by $a_{s}$ and $a_{f}$ to the distinguished variables $s$ and $f$ respectively, to the graph $G$, whose nodes are connected by $b_{s}$ and $b_{f}$ to the distinguished variables $s$ and $f$ respectively.

We show that $G$ is 3-colorable iff MSimp[SOUND,LAV,CQ,CQ] with input $M$ admits a solution. For the "only-if" part, consider the LAV mapping $M_{L}$ consisting of the mapping assertions (8), (9), and (10). If $G$ is 3-colorable, a coloring of the nodes of $G$ with the three colors $r, g, b$ gives us immediately a homomorphism from $q_{G}\left[M_{L}\right]$ to $q_{T}$ in which each variable $x_{i}$ of $q_{G}\left[M_{L}\right]$ is mapped to the variable of $q_{T}$ corresponding to the color assigned to node $n_{i}$. Hence we have that $q_{T} \sqsubseteq q_{G}\left[M_{L}\right]$. For the "if-part", consider a LAV mapping $M_{L}$ such that $q_{s}\left[M_{L}\right] \sqsubseteq q_{t}$ for each mapping assertion $q_{s} \leadsto q_{t}$ in $M$. By the mapping assertions (8), (9), and (10), we have that the queries $M_{L}(t), M_{L}\left(a_{s}\right)$, and $M_{L}\left(a_{f}\right)$, which we assume to have $(x, y)$ as distinguished variables, must include respectively the atoms $e(x, y), b_{s}(x, y)$, and $b_{f}(x, y)$, plus possibly additional atoms containing existentially quantified variables. Note that these existential variables appear in the unfolding $q_{T}\left[M_{L}\right]$. Now, consider a homomorphism $h$ from $q_{G}(s, f)$ to $q_{T}\left[M_{L}\right](s, f)$. Since $s$ and $f$ are distinguished variables, we have that $h(s)=s$ and $h(f)=f$. Suppose that for some variable $x_{i} \in\left\{x_{1}, \ldots, x_{k}\right\}$ of $q_{G}$, we have that $h\left(x_{i}\right)$ is an existential variable $y$ in an additional atom in $q_{T}\left[M_{L}\right]$. Then, since $q_{G}$ contains the atoms $b_{s}\left(s, x_{i}\right)$ and $b_{f}\left(x_{i}, f\right)$, we must have that $q_{T}\left[M_{L}\right]$ contains the atoms $b_{s}(s, y)$ and $b_{f}(y, f)$. This is impossible, since $y$ is an existential variable introduced by the unfolding of $q_{T}$ with $M_{L}$, and hence can appear in the unfolding of just one atom of $q_{T}$. But there is no atom of $q_{T}$ that contains both $s$ and $f$, and that could generate both $b_{s}(s, y)$ and $b_{f}(y, f)$. So, the only possibility for a homomorphism from $q_{G}\left[M_{L}\right]$ to $q_{T}$ is to map each $x_{i}$ of $q_{G}\left[M_{L}\right]$ to one of the variables $r, g$, or $b$. The existence of such a homomorphism implies that $G$ is 3-colorable.

\section{Proof of Lemma 18.}

If $q_{t}=\varepsilon$, we can simply set $M_{L}^{\prime}(a)=\varepsilon$, for each $a \in \Sigma_{s}$. Otherwise, since $q_{s}\left[M_{L}\right] \neq \emptyset$ and $q_{s}\left[M_{L}\right] \subseteq q_{t}$, there exists a nonempty word $a_{1} \cdots a_{k} \in q_{s}$ and a word $w_{1} \cdots w_{k} \in q_{s}\left[M_{L}\right]$ and hence in $q_{t}$, where $w_{j} \in M_{L}\left(a_{j}\right)$. To define the new LAV mapping $M_{L}^{\prime}$, we consider each $a \in \Sigma_{s}$ appearing in $a_{1} \cdots a_{k}$. Notice that $a$ might appear in $a_{1} \cdots a_{k}$ multiple times, and suppose the occurrences of $a$ are $a_{i_{1}}, \ldots, a_{i_{\ell}}$, correspond- 
ing to $w_{i_{1}}, \ldots, w_{i_{\ell}}$. We chose arbitrarily one $w_{i_{j}}$ and set $M_{L}^{\prime}(a)=w_{i_{j}}$. Instead, for each $a \in \Sigma_{s}$ not appearing in $a_{1} \cdots a_{k}$, we set $M_{L}^{\prime}(a)=\emptyset$. Now, $q_{s}\left[M_{L}^{\prime}\right] \neq \emptyset$ by construction, and since $M_{L}^{\prime}(a) \subseteq M_{L}(a)$ for every $a \in \Sigma_{s}$, we have that $q_{s}\left[M_{L}^{\prime}\right] \subseteq q_{s}\left[M_{L}\right] \subseteq q_{t}$.

\section{Proof of Lemma 19}

Let $A_{t}=\left(\Sigma_{t}, S_{t}, s_{t}^{0}, \delta_{t}, F_{t}\right)$. Consider a word $a_{1} \cdots a_{h} \in$ $q_{s}$. If there is one of the $a_{i}$ such that $M_{L}\left(a_{i}\right)=\emptyset$, then $M_{L}\left(a_{1}\right) \cdots M_{L}\left(a_{h}\right)=\emptyset \subseteq q_{t}$. Otherwise, we have that $M_{L}\left(a_{i}\right)=\left\{w^{a_{i}}\right\}$, for $i \in\{1, \ldots, h\}$, and since $w^{a_{1}} \cdots w^{a_{h}} \in$ $q_{s}\left[M_{L}\right] \subseteq q_{t}$, there is a sequence $s_{0}, s_{1}, \ldots, s_{h}$ of states of $A_{t}$ such that $s_{0}=s_{t}^{0}, s_{h} \in F_{t}$, and $s_{i} \in \delta_{t}\left(s_{i-1}, w^{a_{i}}\right)$, for $i \in\{1, \ldots, h\}$. Consider now, for each $i \in\{1, \ldots, h\}$, a word $w_{i}^{\prime} \in M_{L}^{\prime}\left(a_{i}\right)=\left[w^{a_{i}}\right]_{A_{t}}$. Making use of the characterization of $\left[w^{a_{i}}\right]_{A_{t}}$ in terms of a binary relation over $S_{t}$, we have for each word in $\left[w^{a_{i}}\right]_{A_{t}}$, and in particular for $w_{i}^{\prime}$, that $s_{i} \in \delta_{t}\left(s_{i-1}, w_{i}^{\prime}\right)$. Hence, $s_{h} \in \delta_{t}\left(s_{o}^{t}, w_{1}^{\prime} \cdots w_{h}^{\prime}\right)$ and $w_{1}^{\prime} \cdots w_{h}^{\prime} \in q_{t}$. 